\title{
A Nonsmooth, Nonconvex Model of Optimal Growth
}

\author{
Takashi Kamihigashi* \\ RIEB \\ Kobe University \\ tkamihig@rieb.kobe-u.ac.jp
}

\author{
Santanu Roy \\ Department of Economics \\ Southern Methodist University \\ sroy@smu.edu
}

August 11, 2003

\begin{abstract}
This paper analyzes the nature of economic dynamics in a one-sector optimal growth model in which the technology is generally nonconvex, nondifferentiable, and discontinuous. The model also allows for irreversible investment and unbounded growth. We provide sufficient conditions for boundedness, extinction (convergence to zero), survival (boundedness away from zero), and unbounded growth. These conditions reveal that boundedness and survival are symmetrical phenomena, so are extinction and unbounded growth. Since many of the conditions are only local, it is possible that extinction occurs from small capital stocks, while unbounded growth occurs from large capital stocks. Despite such nonclassical results and nonclassical features such as nonconvexity and discontinuity, the model behaves much like a classical one as the discount factor approaches unity. In particular, we show that in most cases, if the discount factor is close to one, any optimal path from a given initial capital stock converges to a small neighborhood of what we define as the golden rule capital stock. If this stock is not finite, i.e., if sustainable consumption is maximized at infinity, then as the discount factor approaches one, unbounded growth at least almost occurs.
\end{abstract}

Keywords: Nonconvex, nonsmooth, and discontinuous technology; optimal growth; unbounded growth; extinction; neighborhood turnpike.

*Corresponding author: RIEB, Kobe Univeristy, Rokkodai, Nada, Kobe 657-8501, Japan Phone/Fax: +81-78-803-7015, Email: tkamihig@rieb.kobe-u.ac.jp 


\section{Introduction}

The vast majority of growth models in the economic literature assume smooth technologies. In most cases, smoothness, or differentiability, is assumed purely for analytical convenience. In reality, however, various types of indivisibility and discreteness are present, so that the actual aggregate technology is most likely nonsmooth and even discontinuous. In addition, nonconvexities enter the aggregate technology through numerous sources, such as fixed costs, threshold effects, increasing returns to scale, economies of scope, and depensation in the reproduction of natural resources. Yet, there has been no attempt in the literature to conduct a systematic analysis of an aggregative optimal growth model that admits a nonconvex, nonsmooth, and discontinuous technology. ${ }^{1}$

This paper offers such an analysis by studying the nature of economic dynamics in a one-sector optimal growth model in which the technology is generally nonconvex, nondifferentiable, and discontinuous. The model also allows for irreversible investment and unbounded growth. The main results of the paper are categorized into two groups. The first group provides sufficient conditions for boundedness, extinction (convergence to zero), survival (boundedness away from zero), and unbounded growth. The second group establishes the neighborhood turnpike property of optimal paths as the discount factor approaches one.

One of the contributions of this paper is to fill the gap between two separate strands of the optimal growth literature. The first is the literature on optimal unbounded growth in one-sector models. This literature dates back at least to McFadden (1967, Sec. 6) and Gale and Sutherland (1968), though they were not primarily concerned with conditions for unbounded growth. More recently, Jones and Manuelli (1990) obtained a condition for unbounded growth in a convex one-sector model with multiple capital stocks. ${ }^{2}$ Essentially, however, the literature on optimal unbounded growth in one-sector models has dealt only with convex technologies. ${ }^{3}$

\footnotetext{
${ }^{1}$ Various results are known for nondifferentiable, convex growth models (e.g., McKenzie, 1986). The discrete-choice problems studied in Kamihigashi (2000a, b) have discontinuous features. More relevant works are discussed below.

${ }^{2}$ The analysis was extended to a multisector model by Dolmas (1996). See De Hek and Roy (2001) for results on stochastic models.

${ }^{3}$ A notable exception is Romer (1986, Sec. V.B), who analyzed a one-sector optimal growth model with a special form of increasing returns. The technology in his model,
} 
The implications of nonconvex technologies have been examined in the literature on optimal growth models with nonconcave production functions. The analysis of optimal paths is fairly extensive for the case of an S-shaped production function (Skiba, 1978; Majumda and Mitra, 1982, 1983; Dechert and Nishimura, 1983). ${ }^{4}$ Models with more general production functions were analyzed by Majumdar and Nermuth (1982), Mitra and Ray (1984), and Amir et al. (1991). ${ }^{5}$ Majumdar and Nermuth also allowed for irreversible investment. The literature on nonconvex optimal growth models, however, has ruled out unbounded growth by assuming the existence of a maximum sustainable capital stock. ${ }^{6}$

The optimal growth literature therefore has lacked a comprehensive analysis of nonconvex one-sector models in which unbounded growth is possible. This paper provides such an analysis. Aside from technical conditions required to rule out trivial cases or to ensure the existence of optimal paths, we only assume that the utility function is strictly concave, that the production function is strictly increasing, and that the lower bound on next period's capital is nondecreasing in current capital. The last assumption allows for a general form of irreversible investment, though it is trivially satisfied in the standard case in which the only lower bound on capital is given by the nonnegativity constraint.

Another contribution of this paper is to provide the first systematic analysis of an optimal growth model in which the technology is generally nonconvex, nondifferentiable, and discontinuous. In terms of generality, the stationary framework used by Mitra and Ray (1984) and Amir et al. (1991) is the closest to ours. In their framework, the production function is only required to be continuous and strictly increasing, though discontinuity, irreversible investment, and unbounded growth are ruled out. Amir et al. (1991) showed the monotonicity of the value function and the optimal consumption policy correspondence with respect to changes in the discount factor. These comparative statics properties are not considered in this paper. Mitra and

however, does not take the standard form.

${ }^{4}$ Early contributions to this literature are studies of optimal management of fisheries and other renewable resources (e.g., Clark, 1971).

${ }^{5}$ The discrete-time models mentioned in this paragraph are discussed in detail at the end of Section 2.

${ }^{6}$ Stochastic models with nonconvex technologies (e.g., Majumdar et al., 1989; Joshi, 1997) are distinct from deterministic ones in that the effect of exogenous shocks, rather than that of initial conditions, essentially determines the long run dynamics. 
Ray (1984) established the monotonicity of optimal paths and the existence of a nonzero steady state. This monotonicity property, which carries over to our model, is our point of departure. We also extend their result concerning the existence of a steady state to our setting as a consequence of one of our lemmas. ${ }^{7}$

The generality of our model poses several technical challenges. The absence of differentiability makes the standard Euler equation invalid. The discontinuity of the technology implies that the value function is generally discontinuous, and that the optimal policy correspondence is generally not upper hemi-continuous. The irreversibility of investment implies that the value function is not necessarily increasing. These difficulties make various familiar techniques inapplicable, but, for this very reason, help gain a deeper insight into the fundamental mechanisms of economic dynamics.

There are four essential tools for overcoming these difficulties. The first is Dechert and Nishimura's (1983) monotonicity argument. In fact, we impose only the very minimum set of assumptions under which their argument works. The second tool is what we call the gain function, which measures discounted net returns on investment. This function has been used in the literature only to analyze the properties of steady states. We show that optimal paths never move in a direction in which higher discounted net returns on investment, or higher "gains," will never be available. This result helps determine the direction in which an optimal path possibly moves. The third tool is our finding that a bounded optimal path converges to an optimal steady state despite the discontinuity of the technology. This is a nontrivial result since the discontinuity of the technology implies the discontinuity of the value function. The forth tool is generalized one-sided derivatives (called Dini derivatives) that are well-defined even for nondifferentiable or discontinuous functions. ${ }^{8}$ Using these derivatives, we derive Euler inequalities instead of an Euler equation, and obtain necessary conditions for a steady state. With these four tools, our general one-sector model can be handled in a rather standard way in many cases.

An interesting feature of the model that our analysis reveals is symmetry

\footnotetext{
${ }^{7}$ Amir et al. (1991) showed other comparative statics results for the finite-horizon case. We only consider the infinite-horizon case. Mitra and Ray (1984) showed a general stability result for the case in which utility is only concave. There is no corresponding result in this paper since we assume strict concavity throughout.

${ }^{8}$ Dini derivatives were used in Kamihigashi (2003) to obtain transversality conditions for general stochastic problems.
} 
between downward and upward dynamics. Our results show that boundedness and survival are completely symmetrical phenomena, so are extinction and unbounded growth. This symmetry has not been recognized in the literature on nonconvex optimal growth models, which has assumed away unbounded growth.

The symmetry between downward and upward dynamics is accentuated in the first group of our main results, which can be roughly summarized as follows. Growth never occurs if higher gains will never be available along any increasing path. Growth never occurs from small capital stocks if higher gains will not be available for a very long time along any increasing path, and if marginal utility goes to infinity as consumption goes to zero. Likewise, decline never occurs if higher gains will never be available along any decreasing path. Decline never occurs from large capital stocks if higher gains will not be available for a very long time along any decreasing path, and if marginal utility goes to zero relatively fast as consumption goes to infinity. These results and the necessary conditions for a steady state derived from Euler inequalities are used to obtain conditions for extinction and unbounded growth. Extinction occurs if an optimal path is bounded and if there is no nonzero steady state to which it can converge. Likewise, unbounded growth occurs if an optimal path is bounded away from zero and if there is no steady state to which it can converge. Since many of our conditions are only local, it is possible, for example, that unbounded growth occurs from large capital stocks, while extinction occurs from small capital stocks, i.e., the rich get richer and the poor get poorer.

Despite such nonclassical results and nonclassical features such as nonconvexity and discontinuity, the model behaves much like a classical one as the discount factor approaches unity. We show this by establishing several neighborhood turnpike results. The neighborhood turnpike property is well-known for convex models (e.g., McKenzie, 1982, 1986; Yano, 1984; Montrucchio, 1994, 1995; Guerrero-Lechtenberg, 2000). It is the property that as the discount factor approaches one, any optimal path from a given initial capital stock "converges" to a small neighborhood of the golden rule capital stock, which is defined as the unique steady state in the undiscounted case. Since this definition does not work in our nonconvex model, we define the gold rule capital stock as the smallest capital stock that achieves maximum sustainable consumption. We show that the neighborhood turnpike property holds if the initial capital stock is less than the golden rule level. If the latter is not finite, i.e., if sustainable consumption is maximized at infinity, then 
as the discount factor approaches one, unbounded growth at least almost occurs: an optimal path either grows unboundedly or converges to an arbitrarily large capital stock. We also show that the neighborhood turnpike property holds regardless of the initial capital stock if irreversible investment is ruled out, or if there is a unique capital stock that achieves maximum sustainable consumption. Moreover, we argue that the neighborhood turnpike property holds rather generically.

An immediate consequence of our results is the turnpike theorem shown by Majumdar and Nermuth (1982) for a special case of our model that assumes, among other things, differentiability and the existence of a unique steady state and a maximum sustainable capital stock. Their result has been the most general turnpike theorem on nonconvex optimal growth models. ${ }^{9}$ Our contribution here is to show that the neighborhood turnpike property holds even when unbounded growth is possible and the technology is nondifferentiable and discontinuous.

The rest of the paper is organized as follows. Section 2 presents the model along with the assumptions that are maintained throughout the paper. Section 3 develops the essential tools discussed above and shows some results of independent interest. Sections 4, 5, 6, and 7 give conditions for boundedness, extinction, survival, and unbounded growth, respectively. Section 8 establishes several neighborhood turnpike results. Section 9 concludes the paper. Longer proofs are relegated to the appendices.

\section{The Model}

Consider the following maximization problem:

$$
\begin{aligned}
\max _{\left\{c_{t}, x_{t}\right\}_{t=0}^{\infty}} & \sum_{t=0}^{\infty} \delta^{t} u\left(c_{t}\right) \\
\text { s.t. } & \forall t \in \mathbb{Z}_{+}, c_{t}+x_{t+1}=f\left(x_{t}\right) \\
& c_{t} \geq 0 \\
& x_{t+1} \geq r\left(x_{t}\right) \\
& x_{0} \text { given, }
\end{aligned}
$$

\footnotetext{
${ }^{9} \mathrm{~A}$ turnpike theorem for the S-shaped case was shown by Majumdar and Mitra (1982) and Dechert and Nishimura (1983).
} 
where $c_{t}$ is consumption in period $t, x_{t}$ is the capital stock at the beginning of period $t, \delta$ is the discount factor, $u$ is the utility function, $f$ is the production function, and $r\left(x_{t}\right)$ is the lower bound on $x_{t+1}$.

Except for (2.4), the structure of the model is that of a standard onesector growth model. In the standard case, $r(x)=0$ for all $x \geq 0$, and $f(x)$ can be written as

$$
f(x)=\tilde{f}(x)+(1-d) x
$$

for some function $\tilde{f}$ and constant $d$, where $\tilde{f}$ is the net production function and $d$ is the depreciation rate (possibly equal to one). In models with irreversible investment, it is typically assumed that $r(x)=(1-d) x$ and $f$ satisfies (2.6). These are special cases of our general formulation. For convenience, we interpret $r\left(x_{t}\right)$ as the depreciated capital stock at the end of period $t$, and call (2.4) the irreversibility constraint and $r(x)$ the depreciation function. In this interpretation, (2.4) means that capital cannot be decreased below its depreciated level. Our formulation allows for nonlinear depreciation, which seems natural but has not received attention in the literature.

We use the following standard definitions. A path $\left\{c_{t}, x_{t}\right\}_{t=0}^{\infty}$ is feasible if it satisfies (2.2)-(2.4). A capital path $\left\{x_{t}\right\}$ is feasible if there is a consumption path $\left\{c_{t}\right\}$ such that $\left\{c_{t}, x_{t}\right\}$ is feasible. A path from $x_{0}$ is a path $\left\{c_{t}^{\prime}, x_{t}^{\prime}\right\}$ such that $x_{0}^{\prime}=x_{0}$. A capital path from $x_{0}$ is defined similarly. A feasible path $\left\{c_{t}, x_{t}\right\}$ is optimal (from $x$ ) if it solves the maximization problem (2.1)-(2.5) (with $x_{0}=x$ ). A feasible capital path $\left\{x_{t}\right\}$ is optimal (from $x$ ) if there is a consumption path $\left\{c_{t}\right\}$ such that $\left\{c_{t}, x_{t}\right\}$ is optimal (from $x$ ). A stationary (capital) path is a constant feasible (capital) path. A pair $(c, x)$ is a steady state if the stationary path $\left\{c_{t}, x_{t}\right\}$ such that $c_{t}=c$ and $x_{t}=x$ for all $t \in \mathbb{Z}_{+}$ is optimal. A capital stock $x \geq 0$ is a steady state if $(c, x)$ is a steady state for some $c \geq 0$.

Aside from technical conditions required to rule out trivial cases or to ensure the existence of optimal paths, we only assume that $u$ is strictly increasing and strictly concave, that $f$ is strictly increasing, and that $r$ is nondecreasing. The precise assumptions are stated and discussed in what follows. They are maintained throughout this paper.

Assumption 2.1. (i) $u: \mathbb{R}_{+} \rightarrow[-\infty, \infty)$ is strictly increasing and strictly concave. (ii) $\lim _{c \downarrow 0} u(c)=u(0) \in[-\infty, \infty)$. (iii) $\delta \in(0,1)$.

The utility function $u$ is not required to be differentiable. Since the case 
$u(0)=-\infty$ is permitted, $u$ can be logarithmic or, more generally, of the CRRA class.

Assumption 2.2. (i) $f: \mathbb{R}_{+} \rightarrow \mathbb{R}_{+}$is strictly increasing and upper semicontinuous. (ii) $f(0)=0$.

The production function $f$ is not required to be continuous or differentiable. Upper semicontinuity, which is essentially a normalization for monotone functions, is assumed here to ensure the existence of optimal paths.

To state our assumption on the depreciation function $r$, for $h: \mathbb{R}_{+} \rightarrow \mathbb{R}$, we define

$$
\begin{aligned}
& h_{-}(x)=\lim _{y \uparrow x} h(y), \\
& h_{+}(x)=\lim _{y \downarrow x} h(y),
\end{aligned}
$$

provided that the right-hand sides are well-defined. Any nondecreasing function $h$ clearly satisfies $h_{-}(x) \leq h(x) \leq h_{+}(x)$.

Assumption 2.3. (i) $r: \mathbb{R}_{+} \rightarrow \mathbb{R}_{+}$is nondecreasing and lower semicontinuous. (ii) $\forall x>0, r_{+}(x)<x$ and $r(x)<f(x)$.

Like the production function $f$, the depreciation function $r$ is not required to be differentiable or continuous. Lower semicontinuity, which is essentially a normalization for monotone functions, is assumed here to ensure the existence of optimal paths. The inequality $r_{+}(x)<x$ basically means that the irreversibility constraint (2.4) is never binding at a steady state. It is easy to see that if $r_{+}(x) \geq x$ for some $x>0$, and if $r$ is strictly increasing, then any feasible capital path from $x_{0}>x$ is bounded below by $x$. Such a technological restriction on feasible paths is ruled out here. The inequality $r(x)<f(x)$ means that strictly positive consumption is feasible at $x$. This is necessary for the maximization problem (2.1)-(2.5) to make sense in the case $u(0)=-\infty$. Assumption 2.3 is satisfied if $r(x)=0$ for all $x>0$, or if $f$ satisfies $(2.6)$ with $d \in(0,1], r(x)=(1-d) x$, and $\tilde{f}(x)>0$ for all $x>0$.

Assumption 2.4. $\forall x>0$, there exists a feasible path $\left\{c_{t}, x_{t}\right\}$ from $x$ such that $\sum_{t=0}^{\infty} \delta^{t} u\left(c_{t}\right)>-\infty$.

This assumption is trivially satisfied if $u$ is bounded below. The assumption is required for the maximization problem (2.1)-(2.5) to make sense. 
Assumption 2.5. $\forall x>0, \sum_{t=0}^{\infty} \delta^{t} u\left(f^{t}(x)\right)<\infty .{ }^{10}$

The only role of this assumption is to ensure the existence of optimal paths and the upper semicontinuity of the value function. It is satisfied, for example, if $u$ is bounded above, or if $u(c)=\ln (c)$ and $f(x)=A x$ for some constant $A>0$. In general Assumption 2.5 is a joint restriction on $u, f$, and $\delta$, and there are various other cases in which it is satisfied.

Many of the conditions stated above are assumed only to ensure the existence of optimal paths. Their roles can be better understood by considering the value function for the maximization problem (2.1)-(2.5):

$$
v\left(x_{0}\right)=\sup _{\left\{x_{t}\right\}_{t=0}^{\infty}} \sum_{t=0}^{\infty} \delta^{t} u\left(f\left(x_{t}\right)-x_{t+1}\right),
$$

where the supremum is taken all over feasible capital paths from $x_{0}$, i.e., capital paths $\left\{x_{t}\right\}$ from $x_{0}$ such that $r\left(x_{t}\right) \leq x_{t+1} \leq f\left(x_{t}\right)$ for all $t \in \mathbb{Z}_{+}$. The set of feasible capital paths from any $x_{0} \geq 0$ is nonempty by Assumption 2.3. Assumptions 2.1 and 2.2 imply that $u\left(f\left(x_{t}\right)-x_{t+1}\right)$ is upper semicontinuous in $\left(x_{t}, x_{t+1}\right)$. Assumptions 2.2 and 2.3 imply that the set $\left\{\left(x_{t}, x_{t+1}\right) \mid r\left(x_{t}\right) \leq\right.$ $\left.x_{t+1} \leq f\left(x_{t}\right)\right\}$ is closed in $\mathbb{R}^{2}$. Assumptions 2.4 and 2.5 ensure $-\infty<v(x)<$ $\infty$ for all $x>0$. Hence the existence of an optimal path from any initial capital stock $x_{0} \geq 0$ is guaranteed by a standard argument (e.g., Ekeland and Scheinkman, 1986, Proposition 4.1).

Various special cases of our model have been analyzed in the literature. In the framework adopted by Majumdar and Mitra (1982) and Dechert and Nishimura (1983), $u$ and $f$ are twice continuously differentiable, $u^{\prime}(0)=\infty$, $f$ is S-shaped, $r(x)=0$ for all $x \geq 0$, and there is a maximum sustainable capital stock, i.e.,

$$
\exists \underline{x}>0, \forall x>\underline{x}, \quad f(x)<x .
$$

This assumption is common to existing studies on nonconvex one-sector optimal growth models. In Majumdar and Nermuth's (1982, Sec. 3) framework, $u$ and $f$ are also twice continuously differentiable with $u^{\prime}(0)=\infty$, and for some $d \in(0,1], r(x)=(1-d) x$ and $f^{\prime}(x)>(1-d)$ for all $x>0$. In Mitra and Ray's (1984, Sec. 5) framework, $f$ is continuous and $r(x)=0$ for all $x \geq 0$. The infinite-horizon case of the model studied by Amir et al. (1991)

$$
{ }^{10} f^{2}(x)=f(f(x)), f^{3}(x)=f(f(f(x))), \text { etc. }
$$


is another special case of our model in which $f$ is continuous and $r(x)=0$ for all $x \geq 0$. Many of the arguments used in the literature apply to our general model as well. We borrow some of them in the next section.

\section{$3 \quad$ Fundamental Properties}

This section establishes fundamental properties of optimal paths. In particular, we show that an optimal capital path is monotone, that a bounded optimal path converges to a steady state, and that an optimal capital path never moves in a direction in which higher gains, or higher discounted net returns on investment, will never be available. We also establish the almost everywhere uniqueness of the policy correspondence, a sufficient condition for the existence of a nonzero steady state, and necessary conditions for a steady state. Many of the results here become essential tools in our subsequent analysis. Some of them are of independent interest.

\subsection{Monotonicity and Convergence}

The Bellman equation for the maximization problem (2.1)-(2.5) is given by

$$
v\left(x_{t}\right)=\max _{r\left(x_{t}\right) \leq x_{t+1} \leq f\left(x_{t}\right)}\left\{u\left(f\left(x_{t}\right)-x_{t+1}\right)+\delta v\left(x_{t+1}\right)\right\} .
$$

Let $K: \mathbb{R}_{+} \rightarrow 2^{\mathbb{R}_{+}}$denote the policy correspondence:

$$
K\left(x_{t}\right)=\left\{x_{t+1} \in\left[r\left(x_{t}\right), f\left(x_{t}\right)\right] \mid v\left(x_{t}\right)=u\left(f\left(x_{t}\right)-x_{t+1}\right)+\delta v\left(x_{t+1}\right)\right\} .
$$

We begin by showing a monotonicity property of $K$.

Lemma 3.1. $\forall x_{0} \geq 0, \forall y_{0}>x_{0}, \forall x_{1} \in K\left(x_{0}\right), \forall y_{1} \in K\left(y_{0}\right), x_{1} \leq y_{1}$.

Proof. Let $0 \leq x_{0}<y_{0}, x_{1} \in K\left(x_{0}\right)$, and $y_{1} \in K\left(y_{0}\right)$. If $x_{0}=0$, we trivially have $x_{1}=0 \leq y_{1}$. Suppose $x_{0}>0$ and $x_{1}>y_{1}$. Then

$$
r\left(x_{0}\right) \leq r\left(y_{0}\right) \leq y_{1}<x_{1} \leq f\left(x_{0}\right)<f\left(y_{0}\right) .
$$

Hence $y_{1}$ is feasible from $x_{0}$, and $x_{1}$ is feasible from $y_{0}$. The rest of the proof is the same as the first paragraph of the proof of Dechert and Nishimura (1983, Theorem 1). ${ }^{11}$

\footnotetext{
${ }^{11}$ Given (3.3), their argument goes through as long as $u$ is strictly concave and $f$ is strictly increasing. Lemma 3.1 can alternatively be shown by applying Topkis (1978, Theorem 6.3).
} 
The next result shows a monotonicity property of optimal capital paths.

Lemma 3.2. Let $\left\{x_{t}\right\}$ be an optimal capital path. Then $\forall t \in \mathbb{Z}_{+}, x_{t} \leq x_{t+1}$ or $\forall t \in \mathbb{Z}_{+}, x_{t} \geq x_{t+1}$.

Proof. See Appendix A.

The same result was shown by Majumdar and Nermuth (1982, Theorem 3.1) and Dechert and Nishimura (1983, Corollary 1) for differentiable cases, and by Mitra and Ray (1984, Lemma 5.2) for a continuous case. Lemma 3.2 follows from an argument used in the working paper version of Mitra and Ray (1984) (Mitra and Ray, 1983).

It is immediate from Lemma 3.2 that every bounded optimal path converges. It is not obvious, however, whether it converges to a steady state (which in our terminology means an optimal steady state). To see why, note that an optimal path $\left\{c_{t}, x_{t}\right\}$ satisfies $v\left(x_{t}\right)=u\left(c_{t}\right)+\delta v\left(x_{t+1}\right)$ for all $t \in \mathbb{Z}_{+}$ by the principle of optimality. If $x_{t} \rightarrow x>0$ and $c_{t} \rightarrow c>0,{ }^{12}$ and if

$$
\lim _{t \uparrow \infty} v\left(x_{t}\right)=v(x)
$$

then $v(x)=u(c)+\delta v(x)$ by the continuity of $u$. This implies that $(c, x)$ is a steady state. But since $f$ and $r$ are not continuous, neither is $v$. Thus (3.4) need not hold if $\left\{x_{t}\right\}$ is an arbitrary convergent sequence. Nevertheless (3.4) can be shown if $\left\{x_{t}\right\}$ is an optimal capital path. A first step toward this is to show the upper semicontinuity of $v$.

Lemma 3.3. $v$ is upper semicontinuous.

Proof. See Appendix A.

Hence $\varlimsup_{t \uparrow \infty} v\left(x_{t}\right) \leq v(x)$ for any convergent capital path $\left\{x_{t}\right\}$ with $x=\lim _{t \uparrow \infty} x_{t}$, in particular, for any bounded optimal capital path. The "reverse" inequality, $v(x) \leq \underline{\lim }_{t \uparrow \infty} v\left(x_{t}\right)$, can be verified by arguing that when $x_{t}$ is close to $x$, the cost of jumping from $x_{t}$ to $x$ is small, so that the "benefit," $v(x)-v\left(x_{t}\right)$, must be likewise small.

Proposition 3.1. Any optimal path that is bounded converges to a steady state.

\footnotetext{
${ }^{12}$ If $x=c=0$, then $(c, x)$ is trivially a steady state.
} 
Proof. See Appendix A.

Except for immediate consequences of our assumptions, upper semicontinuity is the only property of $v$ that we use. In fact, $v$ need not even be monotone. If $r(x)=0$ for all $x \geq 0$, then a higher capital stock is always better because it expands the set of feasible capital stocks for next period. But if $r$ is not constant, then a higher capital stock, which does not always expand the feasible set, is not necessarily better. ${ }^{13}$ The non-monotonicity of $v$ complicates some of our proofs, but only slightly.

The next result is immediate from Lemma 3.1. We state it here for easy reference.

Lemma 3.4. Let $y_{0}>0$. If every optimal capital path from $y_{0}$ is nonincreasing, then every optimal capital path from $x_{0} \in\left[0, y_{0}\right]$ is bounded above by $y_{0}$. Likewise, if every optimal capital path from $y_{0}$ is nondecreasing, then every optimal capital path from $x_{0} \geq y_{0}$ is bounded below by $y_{0}$.

\subsection{Almost Everywhere Uniqueness}

Although optimal paths are in general not unique in this model, the policy correspondence for the Bellman equation (3.1) can be shown to be singlevalued almost everywhere.

Proposition 3.2. The policy correspondence $K$ is single-valued almost everywhere. More specifically, the set $\{x \geq 0 \mid K(x)$ is multivalued $\}$ is countable.

Proof. See Appendix A.

Dechert and Nishimura (1983, p. 352) showed the same result for their model using the envelope theorem and the monotonicity (thus almost everywhere differentiability) of $v$. Their approach does not work in our model, where $v$ is not monotone and $u$ is not differentiable. The proof of Proposition 3.2 uses only the monotonicity property of $K$ stated in Lemma 3.1.

\footnotetext{
${ }^{13}$ For example, suppose $r(x)=0$ for $x \in[0, z]$ and $r(x)=f(x)-\epsilon$ for $x>z$, where $z>0$ and $\epsilon \in(0, f(z))$. Assume $u(0)=0$, and $\delta=0$ for the moment. Then $v(x)=u(f(x))$ for $x \leq z$, but $v(x)=u(\epsilon)<v(f(z))$ for $x>z$. It is easy to see that $v$ has a similar structure for $\delta>0$ close to zero.
} 


\subsection{The Gain Function}

For $x \geq 0$, define

$$
\Gamma(x)=\delta f(x)-x .
$$

We call this function the gain function for the following reason. If $x$ units of capital are invested today, it generates $f(x)$ units of output tomorrow. Thus the discounted net return, or "gain," is $\delta f(x)-x$.

The gain function plays a central role in our analysis. The same function was used by Majumdar and Nermuth (1982, p. 358), Dechert and Nishimura (1983, Lemmas 2, 3), and Mitra and Ray (1984, p. 160, 164) to examine the properties of steady states. We use it for more general purposes.

It is useful to note that for any feasible path $\left\{c_{t}, x_{t}\right\}$ and $T \in \mathbb{N}$,

$$
\begin{aligned}
\sum_{t=0}^{T} \delta^{t} c_{t} & =\sum_{t=0}^{T} \delta^{t}\left[f\left(x_{t}\right)-x_{t+1}\right] \\
& =f\left(x_{0}\right)-x_{1}+\delta\left[f\left(x_{1}\right)-x_{2}\right]+\cdots+\delta^{T}\left[f\left(x_{T}\right)-x_{T+1}\right] \\
& =f\left(x_{0}\right)+\sum_{t=0}^{T-1} \delta^{t} \Gamma\left(x_{t+1}\right)-\delta^{T} x_{T+1} .
\end{aligned}
$$

Thus $\Gamma\left(x_{t+1}\right)$ is the contribution of $x_{t+1}$ to the present discounted value of consumption. The following lemma is shown by generalizing an argument used by Majumdar and Nermuth (1982, p. 358) and Dechert and Nishimura (1983, Lemma 2).

Lemma 3.5. Let $\left\{x_{t}\right\}$ be an optimal capital path that is nonstationary. Then $\exists t \in \mathbb{N}, \Gamma\left(x_{0}\right)<\Gamma\left(x_{t}\right)$.

Proof. See Appendix A.

Therefore a nonstationary optimal capital path must always achieve a higher gain at some point in the future. In other words, optimal capital paths move in a direction in which higher gains will eventually be available. If the highest gain is available at the current capital stock, then it is optimal to stay there forever. Thus the following result is an immediate consequence of Lemma 3.5.

Proposition 3.3. Suppose $\exists \hat{x}>0, \Gamma(\hat{x})=\sup _{x \geq 0} \Gamma(x)$. Then $\hat{x}$ is a steady state. 
As a consequence, we obtain a sufficient set of conditions for the existence of a nonzero steady state.

Proposition 3.4. Suppose (i) $\exists x>0, \Gamma(x) \geq 0$ and (ii) $\varlimsup_{\lim } \uparrow \infty \Gamma(x)<$ $\sup _{x>0} \Gamma(x)$. Then there exists a nonzero steady state.

Proof. Since $f$ is upper semicontinuous, so is $\Gamma$. This together with (ii) implies that $\exists \hat{x} \geq 0, \Gamma(\hat{x})=\sup _{x \geq 0} \Gamma(x) \equiv s$. If $s>0$, then $\hat{x}>0$ since $\Gamma(0)=0$. If $s=0$, then $\hat{x}$ can be chosen to be strictly positive by (i). Thus the conclusion follows by Proposition 3.3.

If $f$ satisfies (i), then it is called $\delta$-productive in Mitra and Ray's (1984, p. 164) terminology. Condition (ii) holds if there is a maximum sustainable capital stock; for then $\Gamma(x)=\delta f(x)-x<f(x)-x<0$ for large $x$ (recall (2.10)). ${ }^{14}$ Hence Proposition 3.4 extends Mitra and Ray (1984, Theorem 4.2) to our general model. ${ }^{15}$ Our argument is similar to theirs, but more direct since we do not consider support prices.

\subsection{Nonsmooth Analysis}

Even in the absence of differentiability, generalized versions of derivatives are available. For $h:(a, b) \rightarrow \mathbb{R}$ with $a<b$, define

$$
\begin{aligned}
& h_{-}^{\prime}(x)=\varliminf_{\epsilon \downarrow 0} \frac{h(x)-h(x-\epsilon)}{\epsilon}, \\
& h_{+}^{\prime}(x)=\varlimsup_{\epsilon \downarrow 0} \frac{h(x+\epsilon)-h(x)}{\epsilon} .
\end{aligned}
$$

These generalized derivatives are called the lower left and the upper right Dini derivative of $h$ evaluated at $x$. They are as informative as the usual onesided derivatives in characterizing the monotonicity properties of an upper semicontinuous function.

\footnotetext{
14 "For large $x "$ means "for all $x$ sufficiently large." Similar remarks apply to similar expressions.

${ }^{15}$ Strictly speaking, since they did not assume the strict concavity of $u$ for their corresponding result, Proposition 3.4 does not generalize their result. But in fact Proposition 3.4 holds even if $u$ is only concave. This is because $\hat{x}$ as given in the proof of Proposition 3.4 is a steady state by (A.14) even if $u$ is only concave.
} 
Lemma 3.6. Let $h:[a, b] \rightarrow \mathbb{R}$ be upper semicontinuous, where $-\infty<a<$ $b<\infty$.

(i) If $h$ is nondecreasing (nonincreasing), then $h_{-}^{\prime} \geq(\leq) 0$ on $(a, b]$ and $h_{+}^{\prime} \geq(\leq) 0$ on $[a, b) .{ }^{16}$

(ii) If $h_{+}^{\prime} \geq 0$ on $[a, b)$, then $h$ is nondecreasing on $[a, b]$.

(iii) If $h_{-}^{\prime} \leq 0$ on $(a, b]$, then $h$ is nonincreasing on $[a, b]$.

Proof. See Giogi and Komlósi (1992, pp. 13-14).

Using the derivatives defined in (3.9) and (3.10), we obtain "Euler inequalities" instead of an Euler equation.

Lemma 3.7. Let $\left\{c_{t}, x_{t}\right\}$ be an optimal path. Let $t \in \mathbb{Z}_{+}$. If $c_{t}>0$ and $x_{t+2}>r_{+}\left(x_{t+1}\right)$, then

$$
u_{-}^{\prime}\left(c_{t}\right) \geq \delta u_{+}^{\prime}\left(c_{t+1}\right) f_{+}^{\prime}\left(x_{t+1}\right) .
$$

If $x_{t+1}>r\left(x_{t}\right)$ and $c_{t+1}>0$, then

$$
u_{+}^{\prime}\left(c_{t}\right) \leq \delta u_{-}^{\prime}\left(c_{t+1}\right) f_{-}^{\prime}\left(x_{t+1}\right) .
$$

Proof. See Appendix A.

If $u$ and $f$ are differentiable, then (3.11) and (3.12) reduce to the Euler equation $u^{\prime}\left(c_{t}\right)=\delta u^{\prime}\left(c_{t+1}\right) f^{\prime}\left(x_{t+1}\right)$. The cost of not assuming differentiability is that the Euler equation must be replaced by the two Euler inequalities above. This cost is rather small here since we use them only to obtain necessary conditions for a steady state. For this purpose, for $x \geq 0$, define

$$
g(x)=f(x)-x
$$

which is the stationary consumption level associated with capital stock $x$. For $x>0$ with $g(x)>0$, define

$$
\Phi(x)=\delta \frac{u_{+}^{\prime}(g(x))}{u_{-}^{\prime}(g(x))} f_{+}^{\prime}(x), \quad \Psi(x)=\delta \frac{u_{-}^{\prime}(g(x))}{u_{+}^{\prime}(g(x))} f_{-}^{\prime}(x) .
$$

If one divides (3.11) through by $u_{-}^{\prime}\left(c_{t}\right)$ and sets $x_{t+1}=x$ and $c_{t}=c_{t+1}=g(x)$, then the resulting right-hand side is $\Phi(x)$. One obtains $\Psi(x)$ similarly. Hence the Euler inequalities (3.11) and (3.12) evaluated at a steady state imply (3.15) below.

\footnotetext{
${ }^{16}$ This part does not require upper semicontinuity.
} 
Lemma 3.8. If $x>0$ is a steady state, then $g(x)>0$ and

$$
\Phi(x) \leq 1 \leq \Psi(x) .
$$

Proof. See Appendix A.

As $u$ is concave, $u_{-}^{\prime} \geq u_{+}^{\prime}$; thus

$$
\forall x>0, \quad \Phi(x) \leq \delta f_{+}^{\prime}(x), \quad \delta f_{-}^{\prime}(x) \leq \Psi(x) .
$$

If $u$ is differentiable, $\Phi(x)=\delta f_{+}^{\prime}(x)$ and $\Psi(x)=\delta f_{-}^{\prime}(x)$. If $u$ and $f$ are differentiable, $\Phi(x)=\Psi(x)=\delta f^{\prime}(x)$. In this case, (3.15) implies $\delta f^{\prime}(x)=$ 1 , a well-known necessary condition for a steady state in the differentiable case. The following result gives useful relationships among the functions $\Phi, \Psi, f_{-}^{\prime}, f_{+}^{\prime}$, and $\Gamma$.

Lemma 3.9. Let $x>0$ and $0 \leq a<b$.

(i) If $\Phi(x)>1$, then $\delta f_{+}^{\prime}(x)>1$.

(ii) $\delta f_{+}^{\prime} \geq 1$ on $[a, b)$ iff $\Gamma$ is nondecreasing on $[a, b]$.

(iii) If $\Psi(x)<1$, then $\delta f_{-}^{\prime}(x)<1$.

(iv) $\delta f_{-}^{\prime} \leq 1$ on $(a, b]$ iff $\Gamma$ is nonincreasing on $[a, b]$.

Proof. Parts (i) and (iii) are immediate from (3.16). Parts (ii) and (iv) hold by Lemma 3.6 because $\Gamma$ is upper semicontinuous, $\Gamma_{+}^{\prime}=\delta f_{+}^{\prime}-1$, and $\Gamma_{-}^{\prime}=\delta f_{-}^{\prime}-1$.

Remark 3.1. If $\exists x>0, f_{-}^{\prime}(x) \leq 1$ or $\Psi(x)<1$, then $f$ is continuous at $x$.

Indeed, any condition implying $f_{-}^{\prime}(x)<\infty$ implies that $f$ is continuous at $x$. To see this, note that if $f$ is discontinuous at some $x>0$, then $f_{-}^{\prime}(x)=\infty$ since $f_{-}(x)<f_{+}(x)$ and $f(x)=f_{+}(x)$ by upper semicontinuity. Some of our conditions for boundedness and extinction imply the local or global continuity of $f$. None of our conditions for survival and unbounded growth implies any continuity of $f$.

\section{Boundedness}

This section offers conditions under which optimal paths are bounded. We begin by stating a result that restricts the gain function $\Gamma$ for large capital stocks. 
Proposition 4.1. Suppose

$$
\exists \underline{x}>0, \forall x>\underline{x}, \quad \Gamma(\underline{x}) \geq \Gamma(x) .
$$

Then every optimal capital path from $x_{0} \in[0, \underline{x}]$ is bounded above by $\underline{x} \cdot{ }^{17}$

Proof. By (4.1) and Lemma 3.5, any optimal capital path from $\underline{x}$ is nonincreasing. Hence the conclusion follows by Lemma 3.4.

Recall from Lemma 3.5 that optimal paths never move in a direction in which higher gains, or higher discounted net returns on investment, will never be available. Condition (4.1) means that gains will never be higher than $\Gamma(\underline{x})$ if capital is increased beyond $\underline{x}$. Under (4.1) therefore it is never optimal to cross $\underline{x}$ from below, which is the conclusion of Proposition 4.1.

A sufficient condition for (4.1) is that $\Gamma(x)$ is nonincreasing for large $x$ or, equivalently, $\delta f_{-}^{\prime}(x) \leq 1$ for large $x$ (recall Lemma 3.9). This condition is used in the following.

Corollary 4.1. Suppose

$$
\exists \underline{x}>0, \forall x>\underline{x}, \quad \delta f_{-}^{\prime}(x) \leq 1 .
$$

Then (i) every optimal capital path is bounded. Furthermore, (ii) every optimal capital path from $x_{0} \geq \underline{x}$ is nonincreasing.

Proof. By (4.2) and Lemma 3.9, $\Gamma$ is nonincreasing on $[\underline{x}, \infty)$. Thus (ii) holds by Lemma 3.5. Proposition 4.1 and (ii) imply (i)

If $u$ and $f$ are differentiable, conclusion (ii) of Corollary 4.1 can be shown using the Euler equation $u^{\prime}\left(c_{t}\right)=\delta u^{\prime}\left(c_{t+1}\right) f^{\prime}\left(x_{t+1}\right)$. Indeed, if $\left\{x_{t}\right\}$ is an optimal capital path from $x_{0} \geq \underline{x}$ that is nondecreasing and nonstationary, then the Euler equation together with (4.2) implies that $\left\{c_{t}\right\}$ is nonincreasing and nonstationary. This leads to a contradiction since the stationary path from $x_{0}$ can be shown to be a better alternative.

Though it may be possible to generalize this approach to the nondifferentiable case, the proof of Corollary 4.1 as well as that of Proposition 4.1 makes no use of the Euler inequalities (3.11) and (3.12). In fact these results rely solely on the shape of the gain function $\Gamma$ through Lemma 3.5.

\footnotetext{
${ }^{17}$ To simplify the statements of our results, we focus on conclusions about capital paths, from which relevant conclusions about consumption paths easily follow.
} 
Recall that Proposition 4.1 restricts $\Gamma$ for large capital stocks. It is based on the idea that growth is not worthwhile if higher gains will never be available along any increasing path. The next result by contrast restricts $\Gamma$ only for small capital stocks. It is based on the idea that growth is not worthwhile if higher gains will not be available for a very long time along any increasing path.

Proposition 4.2. Suppose $\exists z>0$, there exists a sequence $\left\{z_{i}\right\}_{i=1}^{\infty}$ in $(0, z)$ such that

$$
\text { (i) } \lim _{i \uparrow \infty} z_{i}=0, \quad \text { (ii) } \forall i \in \mathbb{N}, \forall x \in\left(z_{i}, z\right], \Gamma\left(z_{i}\right) \geq \Gamma(x) \text {. }
$$

Further suppose

$$
u_{+}^{\prime}(0)=\infty
$$

Then $\exists i \in \mathbb{N}$, every optimal capital path from $x_{0} \in\left(0, z_{i}\right]$ is bounded above by $z_{i}$.

Proof. See Appendix B.

The first hypothesis of Proposition 4.2 holds if $\Gamma$ is nonincreasing on a neighborhood of zero. The second hypothesis, (4.4), is a nonsmooth version of an Inada condition.

Proposition 4.2 can be understood quite intuitively. Condition (4.3) means that if the initial capital stock is small, higher gains will not be available for a very long time along any increasing path. More precisely, if the initial capital stock is $z_{i}$ for some large $i$, it takes a very long time before capital can be increased beyond $z$ to possibly achieve higher gains. If higher gains are not available even there, then it is never optimal to choose an increasing capital path (by Lemma 3.5). However, when no information is available about $\Gamma(x)$ for $x \geq z$, it is potentially optimal to increase capital beyond $z$. This option can be optimal only if the benefit of achieving higher gains in the far future exceeds the cost of sacrificing consumption now. But (4.4) means that sacrificing consumption now is extremely costly when it is already very low due to the small initial capital stock. Hence it is never optimal to increase capital even if higher gains may be available at large capital stocks. 


\section{Extinction}

This section gives conditions under which extinction occurs, i.e., an optimal path converges to zero. Extinction occurs if an optimal capital path is bounded and if there is no nonzero steady state to which it can converge. Nonzero steady states can be ruled out if a necessary condition given by Lemma 3.8 is not met. Conditions for boundedness are given by Propositions 4.1 and 4.2. The following result assumes the condition in Proposition 4.1 .

Proposition 5.1. Assume (4.1). Suppose

$$
\forall x \in(0, \underline{x}], \quad \Psi(x)<1,{ }^{18}
$$

where $\underline{x}$ is given by (4.1). Then every optimal capital path from $x_{0} \in(0, \underline{x}]$ converges to zero.

Proof. By (4.1) and Proposition 4.1, any optimal capital path $\left\{x_{t}\right\}$ from $x_{0} \in(0, \underline{x}]$ is bounded above by $\underline{x}$. By Proposition 3.1, $\left\{x_{t}\right\}$ converges to a steady state. But by (5.1) and Lemma 3.8, there is no steady state in $(0, \underline{x}]$. Hence $\left\{x_{t}\right\}$ converges to zero.

Note from Remark 3.1 that (5.1) implies the continuity of $f$ on $(0, \underline{x}]$. If $f$ is discontinuous at some $x \in(0, \underline{x}]$, then a small decrease from $x$ in capital results in a large loss in output. Thus it is likely that an optimal capital path stays at $x$ instead of going to zero. Condition (5.1) rules out this possibility by ensuring the continuity of $f$ on $(0, \underline{x}]$ as well as the nonexistence of nonzero steady states in $(0, \underline{x}]$. A similar argument applies to (5.2) below. Our conditions for survival and unbounded growth, given in the next two sections, do not imply any continuity of $f$.

If $u$ and $f$ are continuously differentiable, then $\Psi(x)=\delta f^{\prime}(x)$, so that (5.1) holds if $\delta f^{\prime}(0)<1$. This is the condition for local extinction shown by Dechert and Nishimura (1983, Lemma 5). While Proposition 5.1, which assumes (4.1), does not generalize their result, the following does.

Proposition 5.2. Assume (4.4). Suppose

$$
\exists z>0, \forall x \in(0, z], \quad \Psi(x)<1 .
$$

Then $\exists \bar{x} \in(0, z]$, every optimal capital path from $x_{0} \in(0, \bar{x}]$ converges to zero.

\footnotetext{
${ }^{18}$ Recall (3.14) for the definition of $\Psi$.
} 
Proof. Let $\left\{z_{i}\right\}_{i=1}^{\infty}$ be a sequence in $(0, z)$ satisfying (4.3)(i). By (5.2) and Lemma 3.9, $\Gamma$ is nonincreasing on $(0, z]$. Thus $\left\{z_{i}\right\}$ also satisfies (4.3)(ii). By Proposition $4.2, \exists i \in \mathbb{N}$, every optimal capital path from $x_{0} \in\left(0, z_{i}\right]$ is bounded above by $z_{i}$. Let $\bar{x}=z_{i}$. Then every optimal capital path from $x_{0} \in(0, \bar{x}]$ converges to a steady state by Proposition 3.1. By (5.2) and Lemma 3.8 , there is no steady state in $(0, \bar{x}]$. Hence it converges to zero.

As mentioned above, Proposition 5.2 generalizes Dechert and Nishimura (1983, Lemma 5). Their argument cannot easily be extended to our model since it makes extensive use of the Euler equation and their assumptions that $f$ is S-shaped and that there is a maximum sustainable capital stock (which implies that every optimal path converges to a steady state). Olson and Roy (1996, Theorem 6.2) used a more general argument to show local extinction in a slightly different context, but their argument still relies heavily on the Euler equation. Our argument by contrast uses the Euler inequalities (through Lemma 3.8) only to rule out nonzero steady states.

Our last result in this section gives a condition for global extinction.

Proposition 5.3. Suppose

$$
\forall x>0, \quad \Psi(x)<1 .
$$

Then every optimal capital path converges to zero.

Proof. Let $x_{0}>0$. By (5.3) and Lemma 3.9, $\Gamma$ is nonincreasing on $\mathbb{R}_{++}$. Thus (4.1) holds with $\underline{x}=x_{0}$. Hence by Proposition 5.1, any optimal capital path from $x_{0}$ converges to zero.

If $u$ and $f$ are differentiable, (5.3) reduces to the condition that $\delta f^{\prime}(x)<1$ for all $x>0$. This is the condition for global extinction shown by Majumdar and Mitra (1982, p. 122) and Dechert and Nishimura (1983, p. 346) for an S-shaped case. Proposition 5.3 is a direct generalization of their result.

\section{Survival}

This section offers conditions under which survival occurs, i.e., an optimal path is bounded away from zero. The results here are symmetrical to those in Section 4, which gave conditions for boundedness, or "boundedness away from infinity." We begin by stating a result that restricts the gain function $\Gamma$ for small capital stocks. 
Proposition 6.1. Suppose

$$
\exists \bar{x}>0, \forall x \in[0, \bar{x}), \quad \Gamma(x) \leq \Gamma(\bar{x}) .
$$

Then every optimal capital path from $x_{0} \geq \bar{x}$ is bounded below by $\bar{x}$.

Proof. Similar to the proof of Proposition 4.1

Recall once again from Lemma 3.5 that optimal paths never move in a direction in which higher gains, or higher discounted net returns on investment, will never be available. Condition (6.1) means that gains will never be higher than $\Gamma(\bar{x})$ if capital is decreased below $\bar{x}$. Under (6.1) therefore it is never optimal to cross $\bar{x}$ from above, which is the conclusion of Proposition 6.1 .

A sufficient condition for (6.1) is that $\Gamma(x)$ is nondecreasing for small $x$ or, equivalent, $\delta f_{+}^{\prime}(x) \geq 1$ for small $x$ (recall Lemma 3.9). This condition is used in the following.

Corollary 6.1. Suppose

$$
\exists \bar{x}>0, \forall x \in[0, \bar{x}), \quad \delta f_{+}^{\prime}(x) \geq 1 .
$$

Then (i) every optimal capital path is bounded away from zero. Furthermore, (ii) every optimal capital path from $x_{0} \in(0, \bar{x}]$ is nondecreasing.

Proof. Similar to the proof of Corollary 4.1.

Recall that Proposition 6.1 restricts $\Gamma$ for small capital stocks. It is based on the idea that decline is not desirable if higher gains will never be available along any decreasing path. The next result by contrast restricts $\Gamma$ only for large capital stocks. It is based on the idea that decline is not desirable if higher gains will not be available for a very long time along any decreasing path.

Proposition 6.2. Suppose $\exists z>0$, there exists a sequence $\left\{z_{i}\right\}_{i=1}^{\infty}$ in $(z, \infty)$ such that

(i) $\lim _{i \uparrow \infty} z_{i}=\infty$,

(ii) $\forall i \in \mathbb{N}, \forall x \in\left[z, z_{i}\right), \Gamma(x) \leq \Gamma\left(z_{i}\right)$.

Further suppose

$$
\varlimsup_{c \uparrow \infty} u_{+}^{\prime}(c) c<\infty .
$$

Then $\exists i \in \mathbb{N}$, every optimal capital path from $x_{0} \geq z_{i}$ is bounded below by $z_{i}$. 
Proof. See Appendix C.

The first hypothesis of Proposition 6.2 holds if $\Gamma(x)$ is nondecreasing for large $x$. There are various cases in which the second hypothesis, (6.4), holds. For example, it holds if $u$ is bounded above, as implied by the following.

Remark 6.1. If $u$ is bounded above, then

$$
\lim _{c \uparrow \infty} u_{+}^{\prime}(c) c=0 .
$$

Proof. See Appendix C.

Another important case in which (6.4) holds is when $u(c)=\ln c$, which implies $u^{\prime}(c) c=1$ for all $c>0$.

Like Proposition 4.2, Proposition 6.2 can be understood intuitively. Condition (6.3) means that if the initial capital stock is large, higher gains will not be available for a very long time along any decreasing path. More precisely, if the initial capital stock is $z_{i}$ for some large $i$, it takes a very long time before capital can be reduced below $z$ to possibly achieve higher gains. If higher gains are not available even there, then it is never optimal to choose a decreasing capital path (by Lemma 3.5). However, when no information is available about $\Gamma(x)$ for $x<z$, it is potentially optimal to reduce capital below $z$. This option can be optimal only if the benefit of achieving higher gains in the far future exceeds the cost of reducing consumption now in present value (recall (3.6)-(3.8)). But (6.4) means that marginal utility declines relatively fast as consumption grows. In other words, achieving higher gains, or higher consumption, in the far future is not an attractive alternative when high consumption is already feasible due to the large capital stock. Hence it is never optimal to reduce capital even if higher gains may be available at small capital stocks.

\section{Unbounded Growth}

This section gives conditions under which unbounded growth occurs, i.e., an optimal capital path goes to infinity. The results in this section are symmetrical to those in Section 5, which gave conditions for extinction, or convergence to zero. Unbounded growth occurs if an optimal capital path is bounded away from zero and if there is no steady state to which it can 
converge. Steady states can be ruled out if a necessary condition given by Lemma 3.8 is not met. Conditions for boundedness away from zero are given by Propositions 6.1 and 6.2. The following result assumes the condition in Proposition 6.1.

Proposition 7.1. Assume (6.1). Suppose

$$
\forall x \geq \bar{x}, \quad \Phi(x)>1,
$$

where $\bar{x}$ is given by (6.1). Then every optimal capital path from $x_{0} \geq \bar{x}$ goes to infinity.

Proof. Similar to the proof of Proposition 5.1.

If $u$ and $f$ are differentiable, then $\Phi(x)=\delta f^{\prime}(x)$, so that the last inequality in (7.1) reduces to $\delta f^{\prime}(x)>1$.

The following result uses the conditions for survival in Proposition 6.2.

Proposition 7.2. Assume (6.4). Suppose

$$
\exists z>0, \forall x \geq z, \quad \Phi(x)>1 .
$$

Then $\exists \underline{x} \geq z$, every optimal capital path from $x_{0} \geq \underline{x}$ goes to infinity.

Proof. Similar to the proof of Proposition 5.2.

The following result gives a condition for global unbounded growth.

Proposition 7.3. Suppose

$$
\forall x>0, \quad \Phi(x)>1
$$

Then every optimal capital path goes to infinity.

Proof. Similar to the proof of Proposition 5.3.

For a differentiable convex model, Jones and Manuelli (1990, p. 1014) showed that unbounded growth occurs if $\delta f^{\prime}$ is bounded away from $1 .^{19}$ If $u$ and $f$ are differentiable, (7.3) reduces to the condition that $\delta f^{\prime}(x)>1$ for all

\footnotetext{
${ }^{19}$ The sufficient conditions for unbounded growth used by Dolmas (1996, Assumption (P)) and Kaganovich (1998, Assumption 7) reduce to Jones and Manuelli's (1990) condition in the one-sector case with a single capital good.
} 
$x>0$. This condition is necessary for unbounded growth in the differentiable convex case by a standard argument (e.g., Jones and Manuelli, 1997, p. 78). Proposition 7.3 shows that this necessary condition is also sufficient for global unbounded growth even without convexity. ${ }^{20}$

To discuss an implication of convexity, suppose $u$ and $f$ are differentiable and $f$ is concave. Then since $f^{\prime}$ is nonincreasing, either $\delta f^{\prime}(x)>1$ for all $x>0$ or $\delta f^{\prime}(x) \leq 1$ for large $x>0$. This means that unbounded growth either occurs globally (by Proposition 7.3) or never occurs (by Corollary 4.1). Likewise, since either $\delta f^{\prime}(x)<1$ for all $x>0$ or $\delta f^{\prime}(x) \geq 1$ for small $x>0$, extinction either occurs globally (by Proposition 5.3) or never occurs (by Corollary 6.1). Hence unbounded growth and extinction may not coexist in the convex (and differentiable) case. We close this section by pointing out that unbounded growth and extinction may coexist in the nonconvex case.

Corollary 7.1. Assume (4.4), (5.2), (6.4), and (7.2). Then $\exists \bar{x}, \underline{x}>0$ with $\bar{x} \leq \underline{x}$, every optimal capital path from an initial capital stock in $(0, \bar{x}]$ converges to zero, while every optimal capital path from an initial capital stock in $[\underline{x}, \infty)$ goes to infinity.

Proof. Immediate from Propositions 5.2 and 7.2 .

This result may not be particularly surprising in view of Dechert and Nishimura (1983, Theorem 4). Many of their arguments, however, rely heavily on the existence of a maximum sustainable capital stock (recall (2.10)), and thus cannot easily be modified to allow for unbounded growth. Corollary 7.1 suggests the possibility that the rich get richer and the poor get poorer, even without any distortion. ${ }^{21}$

\section{The Neighborhood Turnpike Property}

Despite the nonclassical results shown above and nonclassical features such as nonconvexity and discontinuity, the model behaves much like a classical one as $\delta$ approaches unity. This is shown by establishing several neighborhood

\footnotetext{
${ }^{20}$ One can add the following statement to Propositions 7.1-7.3: the associated consumption path also goes to infinity. This can easily be shown by using (3.11), Lemma C.1, and the fact that $v(x) \geq u(g(x)) /(1-\delta)$ whenever $g(x) \geq 0$.

${ }^{21}$ The corresponding situation in Dechert and Nishimura's model is that the poor get poorer, the modestly rich get richer, and the very rich get less rich.
} 
turnpike results in this section. We show that in most cases, for $\delta$ close to one, any optimal capital path from a given initial stock converges to a small neighborhood of what we define as the gold rule capital stock. ${ }^{22}$ Unlike in $^{2}$ the classical case, this stock is not required to be finite. When it is not finite, unbounded growth at least almost occurs for $\delta$ close to one. Majumdar and Nermuth's (1982) turnpike result is shown to be an immediate consequence of our general results. We also argue that the neighborhood turnpike property holds rather generically. We begin by assuming that maximum sustainable consumption is strictly positive.

Assumption 8.1. $g^{*} \equiv \sup _{x \geq 0} g(x) \in(0, \infty]$.

It is easy to see that if $g^{*} \leq 0$, extinction occurs from every capital stock. ${ }^{23}$ The role of Assumption 8.1 is to rule out this trivial case. Note that we do not require $g^{*}$ to be finite.

We define the golden rule capital stock $x^{*}$ as follows.

$$
x^{*}= \begin{cases}\min \left\{x \geq 0 \mid g(x)=g^{*}\right\} & \text { if } \exists x \geq 0, g(x)=g^{*} \\ \infty & \text { otherwise }\end{cases}
$$

By Assumption 8.1, $x^{*}>0$. The case $x^{*}=\infty$ means that sustainable consumption is maximized at infinity.

This section maintains all the assumptions stated in Section 2 for each $\delta \in(0,1)$. The following is our last assumption.

Assumption 8.2. $\forall x \in\left(0, x^{*}\right], g_{-}(x)>0 .^{24}$

It is easy to see that if $g_{-}(x) \leq 0$, i.e., $f_{-}(x) \leq x$, for some $x \in\left(0, x^{*}\right]$, then no feasible capital path from $x_{0}<x$ can reach $x$. Hence Assumption 8.2 is a minimum requirement for the neighborhood turnpike property to hold globally.

\footnotetext{
${ }^{22}$ This is in fact slightly more general than the standard statement of the neighborhood turnpike theorem. Our version is equivalent to the standard statement under typical hypotheses for the latter.

${ }^{23}$ To see this, suppose $\forall x>0, g(x) \leq 0$. Let $\left\{x_{t}\right\}$ be an optimal capital path from $x_{0}>0$. Then $\left\{x_{t}\right\}$ is nonincreasing since $\forall t \in \mathbb{Z}_{+}, x_{t+1}-x_{t} \leq f\left(x_{t}\right)-x_{t}=g\left(x_{t}\right) \leq 0$. By Lemma 3.8, there is no nonzero steady state. Since $\left\{x_{t}\right\}$ converges to a steady state by Proposition 3.1, it converges to zero.

${ }^{24}$ Recall (2.7) for the definition of $g_{-}$.
} 
For the rest of this section, we take an arbitrary initial capital stock $x_{0} \in \mathbb{R}_{++}$as given. For each $\delta \in(0,1)$, let $\left\{x_{t}^{\delta}\right\}$ be an optimal capital path from $x_{0}$ with the discount factor equal to $\delta$. The neighborhood turnpike property is now expressed as follows.

$$
\lim _{\delta \uparrow 1} \lim _{t \uparrow \infty} x_{t}^{\delta}=x^{*} \cdot{ }^{25}
$$

This equation means that for $\delta$ close to one, $\left\{x_{t}^{\delta}\right\}$ converges to a small neighborhood of $x^{*}$.

The first step to establishing (8.2) is the following result, which is similar to Scheinkman's (1976) "visit lemma."

Lemma 8.1. $\lim _{\delta \uparrow 1} \sup _{t \in \mathbb{Z}_{+}} g\left(x_{t+1}^{\delta}\right)=g^{*}$.

Proof. See Appendix D.

This lemma implies that if $x^{*}$ is the unique maximizer of $g(x)$ (or, more precisely, under (8.6) and (8.7) below), then for $\delta$ close to one, $\left\{x_{t}^{\delta}\right\}$ "visits" a small neighborhood of $x^{*}$ at least once. In general, $\left\{x_{t}^{\delta}\right\}$ approaches some maximizer of $g(x)$ at least once for $\delta$ close to one. The following result shows that if $x_{0} \leq x^{*}$, then $\left\{x_{t}^{\delta}\right\}$ in fact converges to a small neighborhood of $x^{*}$ for $\delta$ close to one.

Proposition 8.1. Suppose $x_{0} \leq x^{*}{ }^{26}$ Then (8.2) holds.

Proof. See Appendix D.

An immediate but important implication of the above result is that if $x^{*}=\infty$, i.e., if sustainable consumption is maximized at infinity, then for $\delta$ close to one, unbounded growth at least almost occurs.

Corollary 8.1. Suppose $x^{*}=\infty$. Then

$$
\lim _{\delta \uparrow \infty t \uparrow \infty} \lim _{t} x_{t}^{\delta}=\infty
$$

Proof. Immediate from Proposition 8.1.

\footnotetext{
${ }^{25}$ Of course, we do not assume that the expression on the left-hand side is well-defined a priori. The same remark applies to Lemma 8.1.

${ }^{26}$ Since $x_{0} \in \mathbb{R}_{++}, x_{0}<x^{*}$ if $x^{*}=\infty$.
} 
This is a "unbounded growth" version of the neighborhood turnpike theorem. Equation (8.3) means that for $\delta$ close to one, unbounded growth at least almost occurs: $\left\{x_{t}^{\delta}\right\}$ either goes to infinity or converges to an arbitrarily large steady state.

The only case that is not covered by Proposition 8.1 is when $x^{*}<\infty$ and $x_{0}>x^{*}$. In this case, (8.2) need not hold, since $\left\{x_{t}^{\delta}\right\}$ could go to infinity or converge to a neighborhood of some $x>x^{*}$ with $g(x)=g^{*}$. However, there are two important cases in which (8.2) is guaranteed to hold even if $x_{0}>x^{*}$. The first case is when it is feasible to jump from $x_{0}$ to $x^{*}$.

Proposition 8.2. Suppose

$$
r\left(x_{0}\right) \leq x^{*} .
$$

Then (8.2) holds.

Proof. See Appendix D.

An obvious sufficient condition for (8.4) is

$$
\forall x \geq 0, \quad r(x)=0,
$$

i.e., the irreversibility constraint is effectively absent. In this case, (8.4) holds regardless of $x_{0}$, so does (8.2).

Note from Assumption 2.3 that (8.4) holds whenever $x_{0} \leq x^{*}$. Suppose $x_{0}>x^{*}$. Then under (8.4), it is suboptimal to stay above $x^{*}$ since current consumption can be increased by jumping down to $x^{*}$, and since the maximum sustainable amount can be consumed every period afterwards by staying at $x^{*}$ forever. If (8.4) does not hold, however, it is infeasible to jump from $x_{0}$ to $x^{*}$, so that a transition to $x^{*}$ could require many periods of low consumption. This is the case, for example, if $r \approx f$ on $\left(x^{*}, x_{0}\right)$.

The following is a simple but useful restatement of Proposition 8.2.

Corollary 8.2. Let $\bar{x}=\sup \left\{x \in \mathbb{R}_{+} \mid r(x) \leq x^{*}\right\}$. If $x_{0} \in(0, \bar{x}]$, then (8.2) holds. ${ }^{27}$

Assumption 2.3 implies $\bar{x}>x^{*}$. Under (8.5), $\bar{x}=\infty$, so that (8.2) holds regardless of $x_{0}$, as mentioned above. Corollary 8.2 makes it clear that there

\footnotetext{
${ }^{27}$ If $\bar{x}<\infty$, then $r(\bar{x}) \leq x^{*}$ by the lower semicontinuity of $r$.
} 
is an interval strictly larger than $\left(0, x^{*}\right]$ on which the neighborhood turnpike property holds.

The second case in which (8.2) holds for $x_{0}>x^{*}$ is when $x^{*}$ is the "unique maximizer" of $g$.

Proposition 8.3. Suppose $x^{*}<\infty$. Suppose

$$
\begin{gathered}
\forall x \in(0, \infty) \backslash\left\{x^{*}\right\}, \quad g(x)<g^{*}, \\
\varlimsup_{x \uparrow \infty} g(x)<g^{*} .
\end{gathered}
$$

Then (8.2) holds.

Proof. See Appendix D.

Assume (8.6). Suppose (i) there is a maximum sustainable capital stock, (ii) there is a neighborhood of zero on which $\Phi>1$ for $\delta$ close to one, and (iii) there is a neighborhood of $x^{*}$ that contains only one steady state $x(\delta)$ for $\delta$ close to one. In this case, (8.7) holds by (i) (recall (2.10)), and Proposition 8.3 along with Lemma 3.8 and Corollary 6.1 implies that for $\delta$ close to one, all optimal capital capital paths from all initial stocks converge to $x(\delta)$. This result was shown by Majumdar and Nermuth (1982, Theorem 3.4) for a special case of our model that assumes, among other things, the differentiability of $u$ and $f .{ }^{28}$

Let us conclude this section by arguing that the neighborhood turnpike property (8.2) is a rather generic one. The results in this section show that (8.2) holds unless (i) $x^{*}<\infty$, (ii) $x^{*}<x_{0}$, (iii) it is infeasible to jump from $x_{0}$ to $x^{*}$, and (iv) $g$ has multiple maximizers, possibly including infinity. The situations in which the neighborhood turnpike property may not hold are unstable since a small perturbation (e.g., in the sup norm sense) to $f$ easily eliminates all but one maximizer of $g$.

Now consider stability of the situations in which the neighborhood turnpike property does hold regardless of the initial capital stock. Corollary 8.1 and Proposition 8.3 show that (8.2) holds if (i) $x^{*}=\infty$ or if (ii) $x^{*}<\infty$ and $g$ has a unique maximizer in the sense of (8.6) and (8.7). If $x^{*}=g^{*}=\infty$, then this remains to be true under small perturbations. On the other hand,

\footnotetext{
${ }^{28}$ The global convergence result shown by Majumdar and Mitra (1982, Theorem 5.5) and Dechert and Nishimura (1983, Theorem 3) for the "mild discount" case can be viewed as a special case of Majumdar and Nermuth's turnpike result in this context.
} 
the case in which $x^{*}=\infty$ and $g^{*}<\infty$ is unstable since $x^{*}$ can be finite after a small perturbation to $f$. But this case is rather exceptional since it implies that the graph of $f(x)$ approaches from below a straight line parallel to the 45 degree line gradually or infinitely often as $x \uparrow \infty$. If $x^{*}<\infty$ and $g$ has a unique maximizer, a small perturbation to $f$ can create multiple maximizers of $g$ in a small neighborhood of $x^{*}$. But this does not affect the neighborhood turnpike property since Lemma 8.1 implies that for $\delta$ close to one, convergence to this neighborhood must occur even after a small perturbation to $f$, and since Corollary 8.2 implies that the new golden rule capital stock is the only "attractor" in the neighborhood.

To summarize, the discussions in the preceding two paragraphs show that the neighborhood turnpike property holds rather generically.

\section{Concluding Remarks}

This paper has analyzed the nature of economic dynamics in a one-sector optimal growth model in which the technology is generally nonconvex, nondifferentiable, and discontinuous. The model also allows for irreversible investment and unbounded growth. One of the contributions of the paper is to fill a gap in the optimal growth literature by analyzing a nonconvex optimal growth model in which unbounded growth is possible. Another contribution is to provide the first systematic analysis of an optimal growth model in which the technology is generally nonconvex, nondifferentiable, and discontinuous. These are properties that the actual aggregate technology is likely to have for various reasons. We have shown sufficient conditions for boundedness, extinction, survival, and unbounded growth. We have also shown that the neighborhood turnpike property holds in most cases.

An interesting feature of the model that our analysis has revealed is symmetry between downward and upward dynamics. In particular, boundedness and survival are symmetrical phenomena, so are extinction and unbounded growth. For example, boundedness occurs if higher gains, or higher discounted net returns on investment, will never be available along any increasing path. Likewise, survival occurs if higher gains will never be available along any decreasing path. All our conditions for boundedness and survival have this symmetrical feature, so do our conditions for extinction and unbounded growth. Since many of these conditions are only local, it is possible, for example, that extinction occurs from small capital stocks, while unbounded 
growth occurs from large capital stocks.

Despite such nonclassical results and nonclassical features such as nonconvexity and discontinuity, the model behaves much like a classical one as the discount factor approaches unity. This has been shown by establishing several neighborhood turnpike results. In most cases, if the discount factor is close to one, any optimal path from a given initial capital stock converges to a small neighborhood of the golden rule capital stock, which is in our definition the smallest capital stock that achieves maximum sustainable consumption. The neighborhood turnpike property holds, for example, if the initial capital stock is smaller than the golden rule level. If the latter is not finite, i.e., if sustainable consumption is maximized at infinity, then as the discount factor approaches one, unbounded growth at least almost occurs: an optimal path either grows unboundedly or converges to an arbitrarily large capital stock. The neighborhood turnpike property also holds if investment is reversible as in the standard case, or if sustainable consumption is maximized at a unique capital stock. We have also argued that the neighborhood turnpike property holds rather generically.

Many of our techniques are directly applicable to other one-dimensional problems with generally nonconvex, nondifferentiable, and discontinuous constraints. The results shown in this paper would provide basic insight into such problems. Though our methods may seem rather specific to one-dimensional cases, some of our arguments are of wider interest. For example, it seems possible to extend the neighborhood turnpike property to multi-sector growth models and other multi-dimensional problems with realistic features such as nonconvexity, nonsmoothness, and discontinuity. The insight gained in this paper would be useful in analyzing such complex problems.

\section{Appendix A Proofs of Section 3 Results}

\section{A.1 Proof of Lemma 3.2}

Lemma A.1. Let $\left\{x_{t}\right\}$ be an optimal capital path. If $x_{0}<x_{1}$, then $\forall t \in$ $\mathbb{Z}_{+}, x_{t} \leq x_{t+1}$. Likewise, if $x_{0}>x_{1}$, then $\forall t \in \mathbb{Z}_{+}, x_{t} \geq x_{t+1}$.

Proof. ${ }^{29}$ Suppose $x_{0}<x_{1}$. Suppose $\exists t \in \mathbb{Z}_{+}, x_{t}>x_{t+1}$. Let $s \in \mathbb{Z}_{+}$be

\footnotetext{
${ }^{29}$ The following argument is due to Mitra and Ray (1983). We include this proof here since their paper, which is the working paper version of Mitra and Ray (1984), is not easily available.
} 
the first $t \in \mathbb{Z}_{+}$with $x_{t}>x_{t+1}$. Note that $x_{s-1} \leq x_{s}$. If $x_{s-1}<x_{s}$, then $x_{s} \leq x_{s+1}$ by Lemma 3.1, a contradiction. Thus $x_{s-1}=x_{s}$. Let $\underline{s} \leq$ $s$ be the first $t \in \mathbb{Z}_{+}$with $x_{t}=x_{s}$. Note that $x_{s-1}<x_{s}$ and that the capital path $\left\{x_{\underline{s}-1}, x_{s}, x_{s+1}, x_{s+2}, \cdots\right\}$ is optimal from $x_{\underline{s}-1}$ by the principle of optimality. Since $x_{\underline{s}-1}<x_{s}$, we must have $x_{s} \leq x_{s+1}$ by Lemma 3.1, again a contradiction. The case $x_{0}>x_{1}$ is similar.

Let us now prove Lemma 3.2. If $\left\{x_{t}\right\}$ is stationary, it trivially has the desired property. Suppose it is nonstationary. Let $s \in \mathbb{Z}_{+}$be the first $t \in \mathbb{Z}_{+}$ with $x_{t} \neq x_{t+1}$. If $x_{s}<x_{s+1}$, then by Lemma A.1, $\forall t \geq s, x_{t} \leq x_{t+1}$; thus $\forall t \in \mathbb{Z}_{+}, x_{t} \leq x_{t+1}$. The case $x_{s}>x_{s+1}$ is similar.

\section{A.2 Proof of Lemma 3.3}

Let $x \geq 0$. Let $\left\{x^{i}\right\}_{i=1}^{\infty}$ be a sequence in $\mathbb{R}_{+}$such that $\lim _{i \uparrow \infty} x^{i}=x$. For $i \in \mathbb{N}$, let $\left\{c_{t}^{i}, x_{t}^{i}\right\}$ be an optimal path from $x^{i}$. Let $y>x$. Without loss of generality, we may assume that $\forall i \in \mathbb{N}, x^{i}<y$. Clearly,

$$
\forall i \in \mathbb{N}, \quad c_{t}^{i} \leq f^{t}(y)
$$

Taking a subsequence, we may assume that $\forall t \in \mathbb{Z}_{+}, x_{t}^{i}$ converges to some $x_{t} \geq 0$ as $i \uparrow \infty$. By the upper semicontinuity of $f, \forall t \in \mathbb{Z}_{+}$,

$$
0 \leq \varlimsup_{i \uparrow \infty} c_{t}^{i}=\varlimsup \lim \left[f\left(x_{t}^{i}\right)-x_{t+1}^{i}\right] \leq f\left(x_{t}\right)-x_{t+1} \equiv c_{t} .
$$

By the lower semicontinuity of $r, \forall t \in \mathbb{Z}_{+}, 0 \leq \overline{\lim }_{i \uparrow \infty}\left(x_{t+1}^{i}-r\left(x_{t}^{i}\right)\right) \leq x_{t+1}-$ $r\left(x_{t}\right)$. Thus the path $\left\{c_{t}, x_{t}\right\}$ is feasible from $x$. By Fatou's lemma, (A.1), and Assumption 2.5,

$$
\begin{aligned}
\varlimsup_{i \uparrow \infty} v\left(x^{i}\right) & =\varlimsup_{i \uparrow \infty} \sum_{t=0}^{\infty} \delta^{t} u\left(c_{t}^{i}\right) \\
& \leq \sum_{t=0}^{\infty} \delta^{t} \varlimsup_{i \uparrow \infty} u\left(c_{t}^{i}\right) \\
& \leq \sum_{t=0}^{\infty} \delta^{t} u\left(c_{t}\right) \leq v(x),
\end{aligned}
$$

where the first inequality in (A.5) uses (A.2), and the second uses the feasibility of $\left\{c_{t}, x_{t}\right\}$. It follows that $v$ is upper semicontinuous. 


\section{A.3 Proof of Proposition 3.1}

Lemma A.2. Let $\left\{x_{t}\right\}$ be a convergent feasible capital path with $x \equiv \lim _{t \uparrow \infty} x_{t} \in$ $(0, \infty)$. Then $\exists \nu \in \mathbb{R}$, for large $t,{ }^{30}$

$$
\frac{u(0)}{1-\delta}<\nu<v\left(x_{t}\right)
$$

Proof. Let $z \in\left(r_{+}(x), x\right)$. This interval is nonempty by Assumption 2.3. Let $\underline{c} \in(0, x-z)$. Since $\lim _{t \uparrow \infty} x_{t}=x$, for large $t$,

$$
r\left(x_{t}\right)<z<z+\underline{c}<x_{t+1} \leq f\left(x_{t}\right) .
$$

Let $t \in \mathbb{Z}_{+}$be large enough to satisfy (A.7). By Assumption 2.4, there is a feasible path $\left\{\hat{c}_{s}, \hat{x}_{s}\right\}$ from $z$ with $\sum_{s=0}^{\infty} \delta^{s} u\left(\hat{c}_{s}\right)>-\infty$. Define $\left\{\tilde{c}_{s}, \tilde{x}_{s}\right\}$ as follows.

$$
\tilde{x}_{0}=x_{t}, \tilde{x}_{1}=z, \tilde{c}_{0}=f\left(x_{t}\right)-z, \quad \forall s \in \mathbb{N}, \tilde{x}_{s+1}=\hat{x}_{s}, \tilde{c}_{s}=\hat{c}_{s-1} .
$$

Then by (A.7), $\tilde{x}_{1} \in\left(r\left(x_{t}\right), f\left(x_{t}\right)\right)$ and $\tilde{c}_{0}>\underline{c}$. Hence by the feasibility of $\left\{\hat{c}_{s}, \hat{x}_{s}\right\}$ from $\tilde{x}_{1}(=z),\left\{\tilde{c}_{s}, \tilde{x}_{s}\right\}$ is feasible from $x_{t}$. Thus

$$
v\left(x_{t}\right) \geq \sum_{s=0}^{\infty} \delta^{s} u\left(\tilde{c}_{s}\right)>u(\underline{c})+\delta \sum_{s=0}^{\infty} \delta^{s} u\left(\hat{c}_{s}\right) \equiv \nu .
$$

Since the last sum is finite and $\underline{c}>0$, (A.6) follows.

Lemma A.3. Let $\left\{c_{t}, x_{t}\right\}$ be an optimal path. If $x \equiv \lim _{t \uparrow \infty} x_{t} \in(0, \infty)$, then $\lim _{t \uparrow \infty} c_{t}>0$.

Proof. Note that $\lim _{t \uparrow \infty} c_{t}$ exists since $\left\{f\left(x_{t}\right)\right\}$ is monotone in $t$ and thus

$$
\lim _{t \uparrow \infty} c_{t}=\lim _{t \uparrow \infty}\left[f\left(x_{t}\right)-x_{t+1}\right]=\lim _{t \uparrow \infty} f\left(x_{t}\right)-x .
$$

Suppose $\lim _{t \uparrow \infty} c_{t}=0$. Note that

$$
\varlimsup_{t \uparrow \infty} v\left(x_{t}\right)=\varlimsup_{t \uparrow \infty} \sum_{i=0}^{\infty} \delta^{i} u\left(c_{t+i}\right) \leq \frac{u(0)}{1-\delta},
$$

where the inequality holds by Fatou's lemma since $\left\{c_{t}\right\}$ is bounded. But (A.11) contradicts Lemma A.2.

\footnotetext{
30 "For large $t$ " means "for all $t$ sufficiently large." Similar remarks apply to similar expressions.
} 
Lemma A.4. Let $\left\{x_{t}\right\}$ be an optimal capital path that is bounded. Suppose $x \equiv \lim _{t \uparrow \infty} x_{t}>0$. Then $\lim _{t \uparrow \infty} v\left(x_{t}\right)=v(x)$.

Proof. Since $\varlimsup_{t \uparrow \infty} v\left(x_{t}\right) \leq v(x)$ by Lemma 3.3, it suffices to show

$$
v(x) \leq \frac{\lim }{t \uparrow \infty} v\left(x_{t}\right) .
$$

Let $\left\{c_{t}\right\}$ be the associated consumption path. Let $c=\lim _{t \uparrow \infty} c_{t}$. By Lemma A.3, $c>0$. Thus by Assumption 2.3 and (A.10), $r_{+}(x)<x<\lim _{t \uparrow \infty} f\left(x_{t}\right)$. Hence for large $t, r\left(x_{t}\right)<x<f\left(x_{t}\right)$. Thus

$$
u\left(f\left(x_{t}\right)-x\right)+\delta v(x) \leq u\left(c_{t}\right)+\delta v\left(x_{t+1}\right) .
$$

Since $\lim _{t \uparrow \infty} u\left(c_{t}\right)=\lim _{t \uparrow \infty} u\left(f\left(x_{t}\right)-x\right)=u(c)$ by (A.10) and continuity, applying $\underline{\lim }_{t \uparrow \infty}$ to (A.13) yields (A.12).

Let us now prove Proposition 3.1. Let $\left\{c_{t}, x_{t}\right\}$ be an optimal path that is bounded. Let $x=\lim _{t \uparrow \infty} x_{t}$ and $c=\lim _{t \uparrow \infty} c_{t}$. By the upper semicontinuity of $f, c+x \leq f(x)$. Note from Assumption 2.3 that $x \geq r(x)$. If $x=0$, then $c=0$, so $(c, x)$ is trivially a steady state. Suppose $x>0$. For $t \in \mathbb{Z}_{+}$, we have $v\left(x_{t}\right)=u\left(c_{t}\right)+\delta v\left(x_{t+1}\right)$. By Lemma A.4 and the continuity of $u$, $v(x)=u(c)+\delta v(x)$. It remains to verify $c+x=f(x)$. But if $c+x<f(x)$, then $v(x) \geq u(f(x)-x)+\delta v(x)>u(c)+\delta v(x)=v(x)$, a contradiction. It follows that $(c, x)$ is a steady state.

\section{A.4 Proof of Proposition 3.2}

Let $k: \mathbb{R}_{+} \rightarrow \mathbb{R}_{+}$be an arbitrary selection from $K$; i.e., $\forall x \geq 0, k(x) \in K(x)$.

Lemma A.5. (i) $k$ is nondecreasing. (ii) If $K$ is multivalued at $x>0$, then $k$ is discontinuous at $x$.

Proof. Lemma 3.1 implies (i). For (ii), suppose $K$ is multivalued at $x_{0}>$ 0, i.e., $\exists x_{1} \geq 0, \exists y_{1}>x_{1},\left\{x_{1}, y_{1}\right\} \subset K\left(x_{0}\right)$. Then by Lemma 3.1, $\forall x \in$ $\left[0, x_{0}\right), k(x) \leq x_{1}$. Thus $k_{-}\left(x_{0}\right) \leq x_{1}$. On the other hand, $\forall x>x_{0}, k(x) \geq y_{1}$. Thus $k_{+}\left(x_{0}\right) \geq y_{1}>x_{1} \geq k_{-}\left(x_{0}\right)$, i.e., $k$ is discontinuous at $x_{0}$.

Let us now prove Proposition 3.2. By Lemma A.5(i), $k$ is nondecreasing; thus it has only countably many discontinuities. Thus by Lemma A.5(ii), $K$ can be multivalued only at finitely many points. 


\section{A.5 Proof of Lemma 3.5}

Lemma A.6. For any feasible path $\left\{c_{t}, x_{t}\right\}$ with $x_{0}>0$,

$$
\sum_{t=0}^{\infty} \delta^{t} u\left(c_{t}\right) \leq \frac{u\left((1-\delta) f\left(x_{0}\right)+\bar{\Gamma}\right)}{1-\delta}
$$

where $\bar{\Gamma}=\sup _{t \in \mathbb{Z}_{+}} \Gamma\left(x_{t+1}\right)$. The inequality is strict if $\left\{c_{t}\right\}$ is not constant.

Proof. It follows from (3.6)-(3.8) that

$$
\sum_{t=0}^{T} \delta^{t} c_{t} \leq f\left(x_{0}\right)+\sum_{t=0}^{T-1} \delta^{t} \bar{\Gamma}
$$

Hence

$$
\sum_{t=0}^{\infty} \delta^{t} c_{t} \leq f\left(x_{0}\right)+\frac{\bar{\Gamma}}{1-\delta} .
$$

Multiplying through by $1-\delta$ and recalling that $u$ is increasing, we get

$$
u\left((1-\delta) \sum_{t=0}^{\infty} \delta^{t} c_{t}\right) \leq u\left((1-\delta) f\left(x_{0}\right)+\bar{\Gamma}\right)
$$

Applying Jensen's inequality to the left-hand side yields (A.14). Since $u$ is strictly concave, (A.14) holds with strict inequality if $\left\{c_{t}\right\}$ is not constant.

Lemma A.7. Let $\left\{c_{t}, x_{t}\right\}$ be a nonstationary optimal path with $x_{0}>0$ such that

$$
\forall t \in \mathbb{Z}_{+}, \quad \Gamma\left(x_{t+1}\right) \leq \Gamma\left(x_{0}\right) .
$$

Then $\left\{c_{t}\right\}$ is not constant. ${ }^{31}$

Proof. Suppose $\left\{c_{t}\right\}$ is constant. By Lemma A.6 and (A.18),

$$
u\left(c_{0}\right) \leq u\left((1-\delta) f\left(x_{0}\right)+\Gamma\left(x_{0}\right)\right)=u\left(g\left(x_{0}\right)\right),
$$

\footnotetext{
${ }^{31}$ The nonstationarity of $\left\{c_{t}, x_{t}\right\}$ only implies that $\left\{x_{t}\right\}$ is not constant. It is possible that $\left\{c_{t}\right\}$ is constant while $\left\{x_{t}\right\}$ is not constant. On the other hand, if $\left\{x_{t}\right\}$ is constant, $\left\{c_{t}\right\}$ is obviously constant.
} 
where $g$ is defined by (3.13). It follows that $c_{0} \leq g\left(x_{0}\right)$. If $c_{0}<g\left(x_{0}\right)$, then this contradicts optimality since the stationary path from $x_{0}$ is feasible (recall Assumption 2.3(ii)). Suppose $c_{0}=g\left(x_{0}\right)$. Then $x_{1}=f\left(x_{0}\right)-g\left(x_{0}\right)=x_{0}$. Since $\left\{c_{t}\right\}$ is constant, it follows that $\forall t \in \mathbb{N}, x_{t}=x_{0}$, contradicting the nonstationarity of $\left\{c_{t}, x_{t}\right\}$.

Let us now prove Lemma 3.5. Let $\left\{c_{t}\right\}$ be the associated consumption path. Assume (A.18). Since $\left\{x_{t}\right\}$ is nonstationary, $\left\{c_{t}\right\}$ is not constant by Lemma A.7. Thus by Lemma A.6 and (A.18),

$$
\sum_{t=0}^{\infty} \delta^{t} u\left(c_{t}\right)<\frac{u\left((1-\delta) f\left(x_{0}\right)+\Gamma\left(x_{0}\right)\right)}{1-\delta}=\frac{u\left(g\left(x_{0}\right)\right)}{1-\delta} .
$$

This requires $g\left(x_{0}\right)>0$, which together with Assumption 2.3(ii) implies that the stationary path from $x_{0}$ is feasible. But this contradicts the optimality of $\left\{c_{t}, x_{t}\right\}$ by (A.20) again.

\section{A.6 Proof of Lemma 3.7}

Proof. We only prove (3.12). The proof of (3.11) is similar. Suppose

$$
x_{t+1}>r\left(x_{t}\right), \quad c_{t+1}>0 .
$$

If $f$ is not left continuous at $x_{t+1}$, then $f_{-}^{\prime}\left(x_{t+1}\right)=\infty$, so (3.12) trivially follows. Suppose $f$ is left continuous at $x_{t+1}$. Consider increasing $c_{t}$ by $\epsilon$, decreasing $x_{t+1}$ by $\epsilon$, and decreasing $c_{t+1}$ by $\mu(\epsilon) \equiv f\left(x_{t+1}\right)-f\left(x_{t+1}-\epsilon\right)$, while keeping the rest of the path unchanged. By (A.21), this perturbation is feasible for small $\epsilon>0$. For small $\epsilon>0$, by optimality,

$$
u\left(c_{t}+\epsilon\right)+\delta u\left(c_{t+1}-\mu(\epsilon)\right) \leq u\left(c_{t}\right)+\delta u\left(c_{t+1}\right)
$$

Rearranging and dividing through by $\epsilon$, we get

$$
\frac{u\left(c_{t}+\epsilon\right)-u\left(c_{t}\right)}{\epsilon} \leq \delta \frac{u\left(c_{t+1}\right)-u\left(c_{t+1}-\mu(\epsilon)\right)}{\mu(\epsilon)} \frac{\mu(\epsilon)}{\epsilon} .
$$

By concavity the left-hand side is monotone in $\epsilon$, so is $\left[u\left(c_{t+1}\right)-u\left(c_{t+1}-\right.\right.$ $\mu(\epsilon))] / \mu(\epsilon)$. Since $\lim _{\epsilon \downarrow 0} \mu(\epsilon)=0$ by the left continuity of $f$ at $x_{t+1}$, applying $\underline{\lim }_{\epsilon \downarrow 0}$ to both sides of (A.23) yields (3.12). 


\section{A.7 Proof of Lemma 3.8}

If $g(x)<0$, the stationary path from $x$ is not feasible. Thus $g(x) \geq 0$. To verify $g(x)>0$, it suffices to show that there is a feasible path $\left\{c_{t}^{\prime}, x_{t}^{\prime}\right\}$ from $x$ such that $c_{0}^{\prime}>0$ and $\sum_{t=0}^{\infty} \delta^{t} u\left(c_{t}^{\prime}\right)>-\infty$; for this implies that a feasible path along which consumption is zero every period cannot be optimal.

Let $c_{0}^{\prime}=(f(x)-r(x)) / 2>0$ and $x_{1}^{\prime}=f(x)-c_{0}^{\prime}>r(x)$, where the first inequality holds by Assumption 2.3. By Assumption 2.4, there is a feasible path $\left\{\hat{c}_{i}, \hat{x}_{i}\right\}$ from $x_{1}^{\prime}$ with $\sum_{i=0}^{\infty} \delta^{i} u\left(\hat{c}_{i}\right)>-\infty$. For $t \in \mathbb{N}$, let $c_{t}^{\prime}=\hat{c}_{t-1}$ and $x_{t+1}^{\prime}=\hat{x}_{t}$. Then clearly $\left\{c_{t}^{\prime}, x_{t}^{\prime}\right\}$ is feasible and has the desired property. It follows that $g(x)>0$.

From Assumption 2.3 and this, $r_{+}(x)<x<f(x)$. Hence (3.11) and (3.12) hold with $c_{t}=c_{t+1}=g(x)$ and $x_{t+1}=x$. Both inequalities in (3.15) now follow.

\section{Appendix B Proof of Proposition 4.2}

Lemma B.1. Let $\left\{c_{t}, x_{t}\right\}$ be an optimal path satisfying

$$
\text { (i) } g\left(x_{0}\right)>0, \quad \text { (ii) } \exists T \in \mathbb{N}, \forall t \leq T-1, \quad \Gamma\left(x_{t+1}\right) \leq \Gamma\left(x_{0}\right) \text {. }
$$

Then

$$
\delta\left[v\left(x_{T+1}\right)-v\left(x_{0}\right)\right] \geq u_{+}^{\prime}\left(g\left(x_{0}\right)\right)\left(x_{T+1}-x_{0}\right) .
$$

Proof. By (3.6)-(3.8) and (B.1)(ii),

$$
\begin{aligned}
\sum_{t=0}^{T} \delta^{t} c_{t} & \leq f\left(x_{0}\right)+\sum_{t=0}^{T-1} \delta^{t} \Gamma\left(x_{0}\right)-\delta^{T} x_{T+1} \\
& =\sum_{t=0}^{T} \delta^{t}\left[f\left(x_{0}\right)-x_{0}\right]+\delta^{T} x_{0}-\delta^{T} x_{T+1}
\end{aligned}
$$

Since $g\left(x_{0}\right)=f\left(x_{0}\right)-x_{0}($ recall $(3.13))$, it follows that

$$
\sum_{t=0}^{T} \delta^{t}\left(c_{t}-g\left(x_{0}\right)\right) \leq-\delta^{T}\left(x_{T+1}-x_{0}\right) .
$$


Define $\left\{\tilde{x}_{t}\right\}$ as follows: $\tilde{x}_{t}=x_{0}$ for $t \leq T+1$ and $\tilde{x}_{t}=x_{t-T-1}$ for $t \geq T+2$. Clearly $\left\{\tilde{x}_{t}\right\}$ is feasible and $\left\{\tilde{x}_{T+1+i}\right\}_{i=0}^{\infty}\left(=\left\{x_{t}\right\}\right)$ is optimal from $x_{0}$. Thus

$$
\sum_{t=0}^{T} \delta^{t} u\left(g\left(x_{0}\right)\right)+\delta^{T+1} v\left(x_{0}\right) \leq \sum_{t=0}^{T} \delta^{t} u\left(c_{t}\right)+\delta^{T+1} v\left(x_{T+1}\right) .
$$

This rearranges to

$$
\begin{aligned}
-\delta^{T+1}\left[v\left(x_{T+1}\right)-v\left(x_{0}\right)\right] & \leq \sum_{t=0}^{T} \delta^{t}\left[u\left(c_{t}\right)-u\left(g\left(x_{0}\right)\right)\right] \\
& \leq \sum_{t=0}^{T} \delta^{t} u_{+}^{\prime}\left(g\left(x_{0}\right)\right)\left(c_{t}-g\left(x_{0}\right)\right) \\
& \leq-u_{+}^{\prime}\left(g\left(x_{0}\right)\right) \delta^{T}\left(x_{T+1}-x_{0}\right),
\end{aligned}
$$

where (B.8) holds by concavity, and (B.9) by (B.5). Now (B.2) follows.

Lemma B.2. Under the hypotheses of Proposition 4.2, $\exists i \in \mathbb{N}$, every optimal capital path from $z_{i}$ is bounded above by $z$.

Proof. Suppose the conclusion is false. Then $\forall i \in \mathbb{N}$, there is an optimal path $\left\{c_{t}^{i}, x_{t}^{i}\right\}$ from $x_{0}^{i}=z_{i}$ such that $\lim _{t \uparrow \infty} x_{t}^{i}>z$. Let $\underline{z}>0$ be such that

$$
f(\underline{z})<z, \quad \frac{\underline{z}}{1-\delta}<z .
$$

Without loss of generality, assume $\forall i \in \mathbb{N}, x_{0}^{i}<\underline{z}$. Note that

$$
\begin{gathered}
\lim _{i \uparrow \infty} g\left(x_{0}^{i}\right)=0, \\
\forall i \in \mathbb{N}, \quad g\left(x_{0}^{i}\right)=f\left(x_{0}^{i}\right)-x_{0}^{i}>f\left(x_{0}^{i}\right)-x_{t_{i}}^{i} \geq 0,
\end{gathered}
$$

where $t_{i}$ is the first $t$ with $x_{t}^{i}>x_{0}^{i}$.

Let $i \in \mathbb{N}$. Let $T_{i}$ be the first $t \in \mathbb{Z}_{+}$with $x_{t+1}^{i}>z$. Note that

$$
x_{0}^{i}<\underline{z}<x_{T_{i}}^{i} \leq z<x_{T_{i}+1}^{i} \leq f\left(x_{T_{i}}^{i}\right) \leq f(z) .
$$

By Lemma B.1,

$$
\delta v\left(x_{T_{i}+1}^{i}\right) \geq u_{+}^{\prime}\left(g\left(x_{0}^{i}\right)\right)\left(x_{T_{i}+1}^{i}-x_{0}^{i}\right)+\delta v\left(x_{0}^{i}\right) .
$$


Recalling (B.13) and (B.12), we get

$$
m \equiv \delta \max _{y \in[z, f(z)]} v(y) \geq u_{+}^{\prime}\left(g^{i}\right)(z-\underline{z})+\frac{\delta u\left(g^{i}\right)}{1-\delta},
$$

where $g^{i}=g\left(x_{0}^{i}\right)$. By concavity, $u\left(g^{i}\right) \geq u(\underline{z})-u_{+}^{\prime}\left(g^{i}\right)\left(\underline{z}-g^{i}\right) \geq u(\underline{z})-u_{+}^{\prime}\left(g^{i}\right) \underline{z}$. Thus

$$
\begin{aligned}
\infty>m & \geq u_{+}^{\prime}\left(g^{i}\right)(z-\underline{z})+\frac{\delta}{1-\delta}\left[u(\underline{z})-u_{+}^{\prime}\left(g^{i}\right) \underline{z}\right] \\
& =u_{+}^{\prime}\left(g^{i}\right)\left[z-\frac{\underline{z}}{1-\delta}\right]+\frac{\delta u(\underline{z})}{1-\delta} .
\end{aligned}
$$

Since the expression in square brackets is strictly positive by (B.10), and since $\lim _{i \uparrow \infty} u_{+}^{\prime}\left(g^{i}\right)=\infty$ by (B.11) and (4.4), the right-hand side of (B.17) goes to $\infty$ as $i \uparrow \infty$, a contradiction.

Let us now prove Proposition 4.2. By Lemma B.2, $\exists i \in \mathbb{N}$, any optimal capital path from $z_{i}$ is bounded above by $z$. Thus by (4.3) and Lemma 3.5, any optimal capital path from $z_{i}$ is nonincreasing. Thus the conclusion holds by Lemma 3.4.

\section{Appendix C Proofs of Section 6 Results}

\section{C.1 Proof of Remark 6.1}

By concavity, $\forall c, \hat{c} \geq 0, u(c) \leq u(\hat{c})+u_{+}^{\prime}(\hat{c})(c-\hat{c})$. Thus

$$
\forall c, \hat{c} \geq 0, \quad u_{+}^{\prime}(\hat{c}) \hat{c} \leq u(\hat{c})-u(c)+u_{+}^{\prime}(\hat{c}) c .
$$

Since $u$ is bounded above, $\lim _{\hat{c} \uparrow \infty} u_{+}^{\prime}(\hat{c})=0$. It follows that

$$
\forall c \geq 0, \quad \varlimsup_{\hat{c} \uparrow \infty} u_{+}^{\prime}(\hat{c}) \hat{c} \leq \lim _{\hat{c} \uparrow \infty} u(\hat{c})-u(c) .
$$

Applying $\lim _{c \uparrow \infty}$ yields (6.5). 


\section{C.2 Proof of Proposition 6.2}

Lemma C.1. Suppose $\exists z>0$, there exists a sequence $\left\{z_{i}\right\}_{i=1}^{\infty}$ in $(z, \infty)$ satisfying (6.3). Then $\exists \theta>0$,

$$
\exists \underline{i} \in \mathbb{N}, \forall i \geq \underline{i}, \quad z_{i} \leq \theta g\left(z_{i}\right) .
$$

Proof. By (6.3), $\forall i \in \mathbb{N}, \delta f\left(z_{i}\right)-z_{i} \geq \delta f(z)-z$; equivalently,

$$
\forall i \in \mathbb{N}, \quad f\left(z_{i}\right)-z_{i} \geq f(z)-\frac{z}{\delta}+\mu z_{i}
$$

where $\mu=1 / \delta-1$. From (C.4), $\forall i \in \mathbb{N}, g\left(z_{i}\right) / z_{i} \geq[f(z)-z / \delta] / z_{i}+\mu$. Let $\theta>1 / \mu$. Then since $\lim _{i \uparrow \infty} z_{i}=\infty, g\left(z_{i}\right) / z_{i} \geq 1 / \theta$ for large $i$, i.e., (C.3) holds.

Lemma C.2. Under the hypotheses of Proposition 6.2, $\exists i \in \mathbb{N}$, any optimal capital path from $z_{i}$ is bounded below by $z$.

Proof. Suppose the conclusion is false. Then $\forall i \in \mathbb{N}$, there is an optimal path $\left\{c_{t}^{i}, x_{t}^{i}\right\}$ from $x_{0}^{i}=z_{i}$ such that $\lim _{t \uparrow \infty} x_{t}^{i}<z$. Let $\theta>0$ and $\underline{i} \in \mathbb{N}$ be as given by Lemma C.1. Without loss of generality, assume $\underline{i}=1$. Then

$$
\forall i \in \mathbb{N}, \quad x_{0}^{i}<\theta g\left(x_{0}^{i}\right) .
$$

Let $i \in \mathbb{N}$. Let $T_{i}$ be the first $t \in \mathbb{Z}_{+}$with $x_{t+1}^{i}<z$. By Lemmas B.1 and C.1,

$$
\delta\left[v\left(x_{0}^{i}\right)-v\left(x_{T_{i}+1}^{i}\right)\right] \leq u_{+}^{\prime}\left(g^{i}\right)\left(x_{0}^{i}-x_{T_{i}+1}^{i}\right) \leq u_{+}^{\prime}\left(g^{i}\right) \theta g^{i},
$$

where $g^{i}=g\left(x_{0}^{i}\right)$. Since the stationary path from $x_{0}^{i}$ is feasible by (C.5),

$$
\delta\left[\frac{u\left(g^{i}\right)}{1-\delta}-\max _{y \in[0, z]} v(y)\right] \leq \theta u_{+}^{\prime}\left(g^{i}\right) g^{i} .
$$

Suppose $u$ is bounded above. Then by (C.5), the left-hand side tends to

$$
\delta\left[\frac{\sup _{c \geq 0} u(c)}{1-\delta}-\max _{y \in[0, z]} v(y)\right]>0
$$

as $i \uparrow \infty$. On the other hand, by (C.5) and Remark 6.1, the right-hand side of (C.7) goes to zero as $i \uparrow \infty$, a contradiction.

Now suppose $u$ is unbounded above. Then by (C.5), the left-hand side of (C.7) goes to $\infty$ as $i \uparrow \infty$, while by (6.4) the right-hand side is uniformly bounded above for large $i$, again a contradiction. 
Let us now prove Proposition 6.2. By Lemma C.2, $\exists i \in \mathbb{N}$, any optimal capital path from $z_{i}$ is bounded below by $z$. Thus by (6.3) and Lemma 3.5, any optimal capital path from $z_{i}$ is nondecreasing. Thus the conclusion holds by Lemma 3.4.

\section{Appendix D Proofs of Section 8 Results}

\section{D.1 Preliminary Lemmas}

Lemma D.1. (i) $\forall \underline{x}>0, \forall \bar{x} \geq \underline{x}, \inf _{x \in[x, \bar{x}]}(x-r(x))>0$. (ii) $\forall \underline{x} \in$ $\left(0, x^{*}\right), \forall \bar{x} \in\left[\underline{x}, x^{*}\right] \cap[\underline{x}, \infty),{ }^{32} \inf _{x \in[\underline{x}, \bar{x}]} g(x)>0$.

Proof. We prove (ii) first. Suppose $\inf _{x \in[x, \bar{x}]} g(x)=0$. Then there exists a sequence $\left\{x^{i}\right\} \subset[\underline{x}, \bar{x}]$ such that $\lim _{i \uparrow \infty} g\left(x^{i}\right)=0$. Taking a subsequence, we may assume that $\left\{x^{i}\right\}$ converges to some $y \in[\underline{x}, \bar{x}]$. Now we obtain the following contradiction: $0=\lim _{i \uparrow \infty} g\left(x^{i}\right) \geq g_{-}(y)>0$, where the last inequality holds by Assumption 8.2. To prove (i), replace $g(x)$ by $(x-r(x))$ and note from Assumption 2.3(ii) that $y-r_{+}(y)>0$.

Lemma D.2. Let $y \in \mathbb{R}_{++}$be such that $y \leq x^{*}$. Then $\forall x>0$, there exists a feasible path $\left\{c_{t}, x_{t}\right\}$ from $x$ such that

$$
\exists T \in \mathbb{Z}_{+}, \quad \forall t \leq T, c_{t}>0, \quad \forall t \geq T+1, c_{t}=g(y), x_{t}=y .
$$

Proof. Suppose $x \geq y$ first. We construct $\left\{c_{t}, x_{t}\right\}$ recursively as follows:

$$
x_{0}=x, \quad \forall t \in \mathbb{Z}_{+}, x_{t+1}=\max \left\{r\left(x_{t}\right), y\right\}, c_{t}=f\left(x_{t}\right)-x_{t+1} .
$$

Let $a=\inf _{z \in[y, x]}(z-r(z))$. By Lemma D.1, $a>0$. If $\exists t \in \mathbb{Z}_{+}, r\left(x_{t}\right)>y$, then $x_{t+1}-x_{t}=r\left(x_{t}\right)-x_{t} \leq-a$. Thus the inequality $r\left(x_{t}\right)>y$ holds only finitely many times or never holds. Let $T$ be the first $t \in \mathbb{Z}_{+}$such that $r\left(x_{t}\right) \leq y$. If $T \geq 1$, then for $t \leq T-1, c_{t}=f\left(x_{t}\right)-r\left(x_{t}\right)>0$ by Assumption 2.3. We have $x_{T+1}=y$ and $c_{T}=f\left(x_{T}\right)-y \geq f(y)-y=g(y) \geq g_{-}(y)>0$, where the last inequality holds by Assumption 8.2 since $y \leq x^{*}$. For $t \geq T+1, c_{t}=g(y)$. It follows that $\left\{c_{t}, x_{t}\right\}$ is feasible and satisfies (D.1).

Now suppose $x<y$. Let $\bar{c} \equiv \inf _{z \in[x, y]} g(z)$. Since $y \leq x^{*}, \bar{c}>0$ by Lemma D.1. Let $\underline{c} \in(0, \bar{c})$. We construct $\left\{c_{t}, x_{t}\right\}$ recursively as follows:

$$
x_{0}=x, \quad \forall t \in \mathbb{Z}_{+}, x_{t+1}=\min \left\{f\left(x_{t}\right)-\underline{c}, y\right\}, c_{t}=f\left(x_{t}\right)-x_{t+1} .
$$

\footnotetext{
${ }^{32}$ If $x^{*}<\infty$, then $\bar{x} \leq x^{*}$; if $x^{*}=\infty$, then $\bar{x}<x^{*}$.
} 
Note that

$$
\forall t \in \mathbb{Z}_{+}, \quad c_{t}=\max \left\{\underline{c}, f\left(x_{t}\right)-y\right\} \geq \underline{c} .
$$

If $\exists t \in \mathbb{Z}_{+}, f\left(x_{t}\right)-\underline{c}<y$, then $x_{t+1}-x_{t}=f\left(x_{t}\right)-\underline{c}-x_{t}=g\left(x_{t}\right)-\underline{c} \geq \bar{c}-\underline{c}>0$. Thus the inequality $f\left(x_{t}\right)-\underline{c}<y$ holds only finitely many times or never holds. Since $\left\{x_{t}\right\}$ is nondecreasing, the irreversibility constraint (2.4) always holds. It follows that $\left\{c_{t}, x_{t}\right\}$ is feasible and satisfies (D.1).

\section{D.2 Proof of Lemma 8.1}

Lemma D.3. Let $y \in \mathbb{R}_{++}$be such that $y \leq x^{*}$. Then

$$
\underline{\varliminf_{\delta \uparrow 1}} \sup _{t \in \mathbb{Z}_{+}} g\left(x_{t+1}^{\delta}\right) \geq g(y) .
$$

Proof. Let $\eta>0$. Let $\left\{\tilde{c}_{t}, \tilde{x}_{t}\right\}$ be any feasible path from $x_{0}$ with

$$
\sup _{t \in \mathbb{Z}_{+}} g\left(\tilde{x}_{t+1}\right) \leq g(y)-\eta
$$

We show that $\left\{\tilde{c}_{t}, \tilde{x}_{t}\right\}$ is suboptimal for $\delta$ close to one. Since $\forall x \geq 0, \Gamma(x)=$ $\delta f(x)-x \leq f(x)-x=g(x)$, it follows by Lemma A.6 that

$$
\forall \delta \in(0,1), \quad(1-\delta) \sum_{t=0}^{\infty} \delta^{t} u\left(\tilde{c}_{t}\right) \leq u\left((1-\delta) f\left(x_{0}\right)+g(y)-\eta\right) .
$$

By Lemma D.2, there is a feasible path $\left\{c_{t}, x_{t}\right\}$ from $x_{0}$ satisfying (D.1). Hence

$$
\forall \delta \in(0,1), \quad(1-\delta) \sum_{t=0}^{T} \delta^{t} u\left(c_{t}\right)+\delta^{T+1} u(g(y)) \leq(1-\delta) v\left(x_{0}\right) .
$$

Note that $T$ does not depend on $\delta$ and that $v$ implicitly depends on $\delta$. As $\delta \uparrow 1$, the left-hand side of (D.8) goes to $u(g(y))$, while the right-hand side of (D.7) goes to $u(g(y)-\eta)<u(g(y))$. It follows that for $\delta$ close to one, any feasible path $\left\{\tilde{c}_{t}, \tilde{x}_{t}\right\}$ satisfying (D.6) is suboptimal.

Since $\left\{x_{t}^{\delta}\right\}$ is optimal for each $\delta \in(0,1)$, we must have $\sup _{t \in \mathbb{Z}_{+}} g\left(x_{t+1}^{\delta}\right)>$ $g(y)-\eta$ for $\delta$ close to one. Since this is true for any $\eta>0$, (D.5) follows. 
Now we prove Lemma 8.1. By the definition of $g^{*}, \varlimsup_{\lim _{\delta \uparrow 1}} \sup _{t \in \mathbb{Z}_{+}} g\left(x_{t+1}^{\delta}\right) \leq$ $g^{*}$. Thus it suffices to show

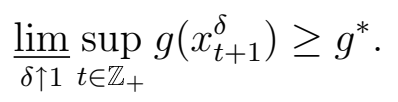

If $x^{*}<\infty$, (D.9) follows from Lemma D.3 with $y=x^{*}$. If $x^{*}=\infty$, applying $\sup _{y \in \mathbb{R}_{++}}$to (D.5) yields (D.9).

\section{D.3 Proof of Proposition 8.1}

Lemma D.4. Suppose $\exists \hat{x}>0, g(\hat{x})=g^{*}$. Then for any $\delta \in(0,1)$, any optimal capital path from $\hat{x}$ is nonincreasing.

Proof. Note that $\forall x \geq \hat{x}, \Gamma(x)=\delta f(x)-x=\delta(f(x)-x)-(1-\delta) x \leq$ $\delta g(\hat{x})-(1-\delta) \hat{x}=\Gamma(\hat{x})$. Thus the conclusion holds by Lemma 3.5.

Lemma D.5. If $x_{0} \leq x^{*}$, then $\varlimsup_{\delta \uparrow 1} \lim _{t \uparrow \infty} x_{t}^{\delta} \leq x^{*}$.

Proof. The inequality is trivial if $x^{*}=\infty$. If $x^{*}<\infty$, it holds by Lemmas 3.4 and D. 4 with $\hat{x}=x^{*}$.

Lemma D.6. If $x_{0}<x^{*}$, then (8.2) holds.

Proof. By Lemma D.5, it suffices to show $\underline{\lim }_{\delta \uparrow 1} \lim _{t \uparrow \infty} x_{t}^{\delta} \geq x^{*}$. Suppose this inequality does not hold; i.e., $\exists \bar{x} \in\left(x_{0}, x^{*}\right)$, there is a sequence $\left\{\delta_{i}\right\}_{i=1}^{\infty}$ in $(0,1)$ with $\lim _{i \uparrow \infty} \delta_{i}=1$ such that $\forall i \in \mathbb{N}, \lim _{t \uparrow \infty} x_{t}^{\delta_{i}} \leq \bar{x}$. By monotonicity, $\forall i \in \mathbb{N}, \forall t \in \mathbb{Z}_{+}, x_{t+1}^{\delta_{i}} \leq \bar{x}$. Thus $\forall i \in \mathbb{N}, \sup _{t \in \mathbb{Z}_{+}} g\left(x_{t+1}^{\delta_{i}}\right) \leq \max _{y \in[0, \bar{x}]} g(y)<$ $g^{*}$, contradicting Lemma 8.1.

Lemma D.7. Let $y \in\left(0, x^{*}\right)$. Then for $\delta$ close to one, every optimal capital path from $y$ is nondecreasing.

Proof. Note that Lemma D.6 holds for any $x_{0} \in\left(0, x^{*}\right)$ and any set of optimal capital paths $\left\{\left\{x_{t}^{\delta}\right\}\right\}_{\delta \in(0,1)}$ from $x_{0}$ such that each $\left\{x_{t}^{\delta}\right\}$ is optimal when the discount factor equals $\delta$. Thus the conclusion follows from monotonicity and Lemma D.6 with $x_{0}=y$.

Lemma D.8. If $x^{*} \leq x_{0}$, then $x^{*} \leq \underline{\lim }_{\delta \uparrow 1} \lim _{t \uparrow \infty} x_{t}^{\delta}$.

Proof. By Lemmas D.7 and 3.4, $\forall y \in\left(0, x^{*}\right), y \leq \underline{\lim }_{\delta \uparrow 1} \lim _{t \uparrow \infty} x_{t}^{\delta}$. Letting $y \uparrow x^{*}$ gives the desired inequality. 
To complete the proof of Proposition 8.1, suppose $x_{0} \leq x^{*}$. If $x_{0}<x^{*}$, then (8.2) holds by Lemma D.6. Suppose $x_{0}=x^{*}$. Then by Lemmas 3.4 and D.4 with $\hat{x}=x^{*}, \overline{\lim }_{\delta \uparrow 1} \lim _{t \uparrow \infty} x_{t}^{\delta} \leq x^{*}$. This together with Lemma D.8 shows (8.2).

\section{D.4 Proof of Proposition 8.2}

Throughout this proof, we assume (8.4). Since Proposition 8.1 covers the case $x_{0} \leq x^{*}$, we also assume $x^{*}<\infty$ and $x^{*}<x_{0}$.

Lemma D.9. Let $z \in\left(x^{*}, x_{0}\right]$. Then for any $\delta \in(0,1)$, there exists no optimal capital path from $z$ that is nondecreasing.

Proof. Let $\left\{c_{t}^{\prime}, x_{t}^{\prime}\right\}$ be a feasible path from $z$ such that $\left\{x_{t}^{\prime}\right\}$ is nondecreasing. Then

$$
\forall t \in \mathbb{Z}_{+}, \quad c_{t}^{\prime}=f\left(x_{t}^{\prime}\right)-x_{t+1}^{\prime} \leq f\left(x_{t}^{\prime}\right)-x_{t}^{\prime}=g\left(x_{t}^{\prime}\right) .
$$

Define $\left\{\tilde{x}_{t}, \tilde{c}_{t}\right\}$ as follows.

$$
\tilde{x}_{0}=z, \tilde{c}_{0}=f(z)-x^{*}, \quad \forall t \geq 1, \tilde{x}_{t}=x^{*}, \tilde{c}_{t}=g\left(x^{*}\right) .
$$

By $(8.4), x^{*}$ is feasible from $z$. Since $x_{1}^{\prime}>x^{*}$,

$$
\tilde{c}_{0}>f(z)-x_{1}^{\prime}=c_{0}^{\prime} \geq 0 .
$$

Thus $\left\{\tilde{c}_{t}, \tilde{x}_{t}\right\}$ is feasible. Since $x^{*}<z, \tilde{c}_{0}>g(z)$. Recalling (D.10) and (D.12), we see that $c_{0}^{\prime}<\tilde{c}_{0}$ and $\forall t \in \mathbb{N}, c_{t}^{\prime} \leq \tilde{c}_{t}$. Since $\left\{\tilde{c}_{t}, \tilde{x}_{t}\right\}$ was arbitrary, it follows that any nondecreasing feasible capital path from $z$ is suboptimal.

Let us now prove Proposition 8.2. By Lemma D.9, $\forall \delta \in(0,1), \lim _{t \uparrow \infty} x_{t}^{\delta} \leq$ $x^{*}$. Thus $\overline{\lim }_{\delta \uparrow 1} \lim _{t \uparrow \infty} x_{t}^{\delta} \leq x^{*}$. This together with Lemma D.8 shows (8.2).

\section{D.5 Proof of Proposition 8.3}

If $x_{0} \leq x^{*}$, (8.2) holds by Proposition 8.1. Suppose $x_{0}>x^{*}$. By Lemma D.8, it suffices to verify $\varlimsup_{\delta \uparrow 1} \lim _{t \uparrow \infty} x_{t}^{\delta} \leq x^{*}$. Suppose $\varlimsup_{\delta \uparrow 1} \lim _{t \uparrow \infty} x_{t}^{\delta}>x^{*}$. Then $\exists \underline{x} \in\left(x^{*}, x_{0}\right)$, there is a sequence $\left\{\delta_{i}\right\}_{i=1}^{\infty}$ in $(0,1)$ with $\lim _{i \uparrow \infty} \delta_{i}=1$ such that $\forall i \in \mathbb{N}, \lim _{t \uparrow \infty} x_{t}^{\delta_{i}} \geq \underline{x}$. By monotonicity, $\forall i \in \mathbb{N}, \forall t \in \mathbb{Z}_{+}, x_{t}^{\delta_{i}} \geq \underline{x}$. Hence $\forall i \in \mathbb{N}, \sup _{t \in \mathbb{Z}_{+}} g\left(x_{t+1}^{\delta_{i}}\right) \leq \sup _{x \geq x} g(x)<g^{*}$, where the last inequality holds by (8.6) and (8.7). But this contradicts Lemma 8.1. 


\section{References}

Amir, R., L.J. Mirman, and W.R. Perkins, 1991, "One-Sector Nonclassical Optimal Growth: Optimality Conditions and Comparative Dynamics," International Economic Review 32, 625-644.

Clark, C.W., 1971, "Economically Optimal Policies for the Utilization of Biologically Renewable Resources," Mathematical Biosciences 12, 245-260.

Dechert, R., and K. Nishimura, 1983, "A Complete Characterization of Optimal Growth Paths in an Aggregated Model with a Non-Concave Production Function," Journal of Economic Theory 31, 332-354.

De Hek, P.A., and S. Roy, 2001, "On Sustained Growth Under Uncertainty," International Economic Review 42, 801-13.

Dolmas, J., 1996, "Endogenous Growth in Multisector Ramsey Models," International Economic Review 37, 403-21.

Ekeland, I., and J.A. Scheinkman, 1986, "Transversality Conditions for Some Infinite Horizon Discrete Time Optimization Problems," Mathematics of Operations Research 11, 216-229.

Gale, D., and W.R. Sutherland, 1968, "Analysis of a One-Good Model of Economic Development," in Mathematics of the Decision Sciences, Part 2, ed. by G. Dantzig and A. Veinott. Providence: American Mathematical Society, pp. 120-136.

Giogi, G., and S. Komlósi, 1992, "Dini Derivatives in Optimization - Part I," Rivista di Matematica per le Scienze Economiche e Sociali 15, 3-30.

Guerrero-Luchtenberg, C.L., 2000, "A Uniform Neighborhood Turnpike Theorem and Applications," Journal of Mathematical Economics 24, 329357.

Jones, L.E., and R.E. Manuelli, 1990, "A Convex Model of Equilibrium Growth: Theory and Policy Implications," Journal of Political Economy 98, 10081038.

Jones, L.E., and R.E. Manuelli, 1997, "The Sources of Growth," Journal of Economic Dynamics and Control 21, 75-114.

Joshi, S., 1997, "Turnpike Theorems in Nonconvex Nonstationary Environments," International Economic Review 38, 225-248.

Kaganovich, M., 1998, "Sustained Endogenous Growth with Decreasing Returns and Heterogeneous Capital," Journal of Economic Dynamics and Control 22, 1575-1603. 
Kamihigashi, T., 2000a, "The Policy Function of a Discrete-Choice Problem is a Random Number Generator," Japanese Economic Review 51, 51-71.

Kamihigashi, T., 2000b, "Indivisible Labor Implies Chaos," Economic Theory $15,585-598$.

Kamihigashi, T., 2003, "Necessity of Transversality Conditions for Stochastic Problems," Journal of Economic Theory 109, 140-149.

Majumdar, M., and T. Mitra, 1982, "Intertemporal Allocation with a NonConvex Technology: The Aggregative Framework," Journal of Economic Theory 27, 101-136

Majumdar, M., and T. Mitra, 1983, "Dynamic Optimization with a Non-Convex Technology: The Case of a Linear Objective Function," Review of Economic Studies 50, 143-151

Majumdar, M., Mitra, T., and Y. Nyarko, 1989, "Dynamic Optimization under Uncertainty: Non-convex Feasible Set", in Joan Robinson and Modern Economic Theory. Hampshire: Macmillan Press, pp. 545-590.

Majumdar, M., and M. Nermuth, 1982, "Dynamic Optimization in Non-Convex Models with Irreversible Investment: Monotonicity and Turnpike Results," Journal of Economics 42, 339-362.

McFadden, D., 1967, "The Evaluation of Development Programmes," Review of Economic Studies 34, 25-50.

McKenzie, L.W., 1982, "A Primary Route to the Turnpike and Liapunov Stability," Journal of Economic Theory 27, 194-209.

McKenzie, L.W., 1986, "Optimal Economic Growth, Turnpike Theorems and Comparative Dynamics," in Handbook of Mathematical Economics, Vol. III, ed. by K.J. Arrow and M.D. Intrilligator. Amsterdam: NorthHolland, pp. 1281-1355.

Mitra, T. and D. Ray, 1983, "Dynamic Optimization on a Non-Convex Feasible Set: Some General Results for Non-Smooth Technologies," Working Paper \#305, Department of Economics, Cornel University.

Mitra, T. and D. Ray, 1984, "Dynamic Optimization on a Non-Convex Feasible Set: Some General Results for Non-Smooth Technologies," Journal of Economics 44, 151-174.

Montrucchio, L., 1994, "The Neighborhood Turnpike Property for ContinuousTime Optimal Growth Models," Ricerche Economiche 48, 213-224. 
Montrucchio, L., 1995, "A New Turnpike Theorem for Discounted Programs," Economic Theory 5, 371-382.

Olson, L.J., and S. Roy, 1996, "On Conservation of Renewable Resources with Stock-Dependent Return and Nonconcave Production," Journal of Economic Theory 70, 133-157.

Romer, P.M., 1986, "Increasing Returns and Long-Run Growth," Journal of Political Economy 94, 1002-1037.

Scheinkman, J.A., 1976, "On Optimal Steady States of n-Sector Growth Models when Utility is Discounted," Journal of Economic Theory 12, 11-30.

Skiba, A., 1978, "Optimal Growth with a Convex-Concave Production Function," Econometrica 46, 527-540.

Topkis, D.M., 1978, "Minimizing a Submodular Function on a Lattice," $O p$ erations Research 26, 305-321.

Yano, M., 1984, "Competitive Equilibria on Turnpikes in a McKenzie Economy, I: A Neighborhood Turnpike Theorem," International Economic Review 25, 695-717. 